\title{
FRUITFULL-like genes regulate flowering time and inflorescence architecture in tomato
}

Xiaobing Jiang ${ }^{a, b}$, Greice Lubini ${ }^{b, c, d}$, José Hernandes-Lopes ${ }^{b, e}$, Kim Rijnsburger ${ }^{a}$, Vera Veltkamp ${ }^{a, b}$, Ruud A. de Maagd ${ }^{b}$, Gerco C. Angenent ${ }^{a, b}$, Marian Bemer ${ }^{a, b}$

a Laboratory of Molecular Biology, Wageningen University \& Research, Droevendaalsesteeg 1, 6708 PB Wageningen, The Netherlands

b Business Unit Bioscience, Wageningen University \& Research, Wageningen, Droevendaalsesteeg 1, 6708 PB Wageningen The Netherlands

' Departamento de Biologia, Faculdade de Filosofia, Ciências e Letras de Ribeirão Preto, Universidade de São Paulo, Ribeirão Preto 14040-901, Brazil

d PPG-Genética, Faculdade de Medicina de Ribeirão Preto, Universidade de São Paulo, Ribeirão Preto 14049-900, Brazil

e Departamento de Botânica, Instituto de Biociências, Universidade de São Paulo, Rua do Matão 277, 05508-090, São Paulo, SP, Brazil

Corresponding author: marian.bemer@wur.nl

The author responsible for distribution of materials integral to the findings presented in this article in accordance with the policy described in the Instructions for Authors (www.plantcell.org) is: Marian Bemer (marian.bemer@wur.nl).

Short title:

Tomato FUL-like genes regulate flowering

ABSTRACT 
The timing of flowering and inflorescence architecture are critical for the reproductive success of tomato, but the gene regulatory networks underlying these traits have not been fully explored. Here we show that the tomato FRUITFULL-like (FUL-like) genes FUL2 and MADS-BOX PROTEIN 20 (MBP20) induce flowering and repress inflorescence branching by promoting floral meristem determinacy. FUL1 fulfils a less prominent role and appears to depend on FUL2 and MBP20 for its upregulation in the inflorescence- and floral meristems. MBP10, the fourth tomato FUL-like gene, has probably lost its function. The tomato FUL-like proteins cannot homodimerize in in vitro assays, but heterodimerize with various other MADS-domain proteins, potentially forming distinct complexes in the transition meristem and floral meristem. Transcriptome analysis of the primary shoot meristems revealed various interesting downstream targets, including four repressors of cytokinin signalling that are upregulated during the vegetative-to-reproductive transition in ful1 ful2 mbp10 mbp20 mutants. FUL2 and MBP20 can also bind in vitro to the upstream regions of these genes, thereby probably directly stimulating cell division in the meristem upon the transition to flowering. The control of inflorescence branching does not occur via the CKXs, but appears regulated by repression of transcription factors such as $T M 3$, APETALA 2b (AP2b) and AP2a.

\section{INTRODUCTION}

The MADS-box transcription factor gene family is involved in almost every developmental process in plants (Smaczniak et al., 2012a), and the members of the angiosperm-specific APETALA1/FRUITFULL (AP1/FUL) subfamily play key roles in flowering and fruit development (Litt and Irish, 2003; McCarthy et al., 2015). In the core eudicots, the AP1/FUL subfamily consists of three clades, euAP1, euFULI and euFULII (Litt and Irish, 2003). The Arabidopsis genome carries four AP1/FUL-clade genes, with $A P 1$ functioning as an A-class gene in the $A B C$ model, promoting perianth identity (Mandel et al., 1992; Theissen and Saedler, 2001) and establishing floral meristem identity and floral initiation (Ferrándiz et al., 2000; Kaufmann et al., 2010). Its lower-expressed paralog CAULIFLOWER (CAL) functions to a large extent redundantly with AP1 (Bowman et al., 1993; Ye et al., 2016). The euFULIl-clade gene AGL79 appears to have a minor function in roots (Gao et al., 2018), whereas the euFULI gene FUL is a pleiotropic gene with important roles in fruit development 
and flowering. FUL regulates many aspects of flowering in Arabidopsis, including flowering time (Ferrándiz et al., 2000; Melzer et al., 2008), repression of inflorescence meristem (IM) identity (together with AP1/CAL; Ferrándiz et al., 2000), inflorescence architecture (Bemer et al., 2017), axillary inflorescence outgrowth (together with SOC1; Karimi et al., 2020) and IM termination (Balanzà et al., 2018). In a wide range of angiosperm species, FUL-like genes regulate fruit development (Gu et al., 1998; Jaakola et al., 2010; Bemer et al., 2012; Pabón-Mora et al., 2012; Zhao et al., 2019) and flowering (Ferrándiz et al., 2000; Berbel et al., 2012; Pabón-Mora et al., 2013; Ping et al., 2014; Jaudal et al., 2015; Jia et al., 2015; Li et al., 2019; Zhang et al., 2021). In the model crop species tomato (Solanum lycopersicum), the euFULI-clade genes Solanum lycopersicum FUL1 (SIFUL1/TM4/TDR4, hereafter called FUL1) and SIFUL2 (FUL2/MBP7, hereafter called FUL2) play important roles in fruit development and ripening (Bemer et al., 2012; Shima et al., 2013; Wang et al., 2019), but flowering phenotypes have not yet been described for these genes, nor for the tomato euFULII-clade genes MADSBOX PROTEIN 10 (MBP10) and MBP20. This is remarkable given the strong upregulation of FUL1, FUL2 and MBP20 expression during the transition from shoot apical meristem (SAM) to inflorescence- and floral meristem (Park et al., 2012).

Flowering is an important agricultural trait in tomato, as both the onset and termination of flowering, as well as inflorescence architecture, determine crop yield. Tomato is also an interesting model species considering its sympodial inflorescence architecture, which is distinct from that of the monopodial Arabidopsis inflorescence. While the Arabidopsis shoot apical meristem (SAM) develops into an inflorescence meristem (IM), which subsequently forms floral meristems (FM) on its flank (indeterminate inflorescence), the tomato SAM is doming to form the transition meristem (TM) that terminates directly into an FM (determinate inflorescence), but forms a new IM on its flank. This iterative process results in a zigzagged inflorescence (Lippman et al., 2008). Moreover, tomato has a compound shoot, which resumes vegetative growth from the axillary meristem of the youngest leaf when the first IM terminates. After activation of the axillary meristem (then called sympodial meristem, SYM), the shoot forms three leaves before terminating again into the first flower of the second inflorescence (Pnueli et al., 1998; Szymkowiak and Irish, 2006; Lippman et al., 2008), upon which a new axillary meristem takes over 
vegetative growth. This process forms the compound shoot, where three leaves and an inflorescence comprise a sympodial unit, a pattern that is endlessly repeated in the wild-type tomato.

The genes that regulate these flowering processes have been very well studied in the indeterminate Arabidopsis inflorescence, where the floral integrators FLOWERING LOCUS T (FT) and SUPPRESSOR OF OVEREXPRESSION OF CONSTANS1 (SOC1) induce the transition to flowering, after which TERMINAL FLOWER 1 (TFL1) determines IM fate by repressing FM genes such as LEAFY (LFY) and AP1 (Sablowski, 2007; Lee and Lee, 2010; Serrano-Mislata et al., 2017; Zhu et al., 2020), while AP1 on its turn represses TFL1, so that clear borders between the FM and IM meristems are achieved (Liu et al., 2013; Goslin et al., 2017). The variation in inflorescence structures of different species can be largely explained by different temporal and spatial expression of flower-repressing TFL homologs and flower-inducing LFY/AP1/FUL homologs (McGarry and Ayre, 2012; Périlleux et al., 2019). In legumes for example, the indeterminate inflorescence does not form FMs on its flank, but secondary IMs, due to repression of the TFL-homolog in these meristems by the euFULII clade proteins VEG1 (pea) or MtFUL1-C (Medicago) (Berbel et al., 2012; Cheng et al., 2018; Zhang et al., 2021). These secondary IMs do form FMs, resulting in a compound inflorescence (Benlloch et al., 2015).

In tomato, SINGLE FLOWER TRUSS (SFT), FALSIFLORA (FA) and MC are essential for the transition to flowering and control of floral meristem identity similar to their orthologs FT, LFY and AP1 in Arabidopsis (Molinero $\square$ Rosales et al., 1999; Molinero-Rosales et al., 2004; Yuste-Lisbona et al., 2016). However, mutants in the TFL-ortholog SELF PRUNING (SP), which is not expressed in the primary SAM/VM, but highly expressed in early axillary meristems (Thouet et al., 2008), loose this growth indeterminacy and terminate their SYMs early, resulting in termination of growth after a few sympodial units (Pnueli et al., 1998). Inflorescence architecture is normal in sp mutants (Pnueli et al., 1998). Instead, tomato inflorescence architecture is influenced by other factors that regulate the establishment or timing of FM maturation/termination (Lippman et al., 2008). Delayed meristem maturation, occurring in the mutants compound inflorescences (s), falsiflora (fa; $F A$ is the ortholog of $L F Y$ ) and anantha (an), induces additional IM formation before the 
determinate FM has matured (Szymkowiak and Irish, 2006; Chae et al., 2008; Lippman et al., 2008; Souer et al., 2008; Park et al., 2012; Soyk et al., 2017), resulting in branched (compound) inflorescences. Conversely, precocious activation of the AN/FA complex leads to early FM induction and thus early flowering (MacAlister et al., 2012). In addition to these factors, several MADS-domain transcription factors function in tomato flowering, mainly in conferring IM or FM identity. In the jointless $(j)$ and macrocalyx $(m c)$ mutants, flowering is delayed and the inflorescence reverts to vegetative growth after a few flowers, probably because the meristems adopt SYM identity instead of FM identity (Szymkowiak and Irish, 2006; Yuste-Lisbona et al., 2016). Mutations in the SEPALLATA-like genes JOINTLESS 2 (J2/SIMBP21), ENHANCER OF JOINTLESS 2 (EJ2/MADS1) and the SOC1-like genes TM3 and SISTER OF TM3 (STM3) affect inflorescence branching through yet uncharacterized mechanisms (Gomez Roldan et al., 2017; Soyk et al., 2017; Soyk et al., 2019; Alonge et al., 2020). Interestingly the tm3 stm3 mutations suppress the enhanced branching phenotype of the j2 ej2 mutant (Alonge et al., 2020), suggesting that these MADS-domain TFs have an opposite function in FM development. Natural mutations or structural variants in several of these MADS-box genes have been important for domestication, either through the regulation of pedicel abscission (MC, J, SIMBP21/J2) or inflorescence architecture (J2, EJ2, TM3, STM3) (Nakano et al., 2012; Liu et al., 2014; Gomez Roldan et al., 2017; Soyk et al., 2019; Alonge et al., 2020). Thus, although tomato inflorescence development differs fundamentally from that of monopodial species such as Arabidopsis, the orthologs of several important Arabidopsis flowering and inflorescence meristem genes are also essential in tomato. However, it is yet unclear if and how the FUL-like genes (SIFUL) function in the tomato flowering regulatory network.

Here, we investigated the developmental roles of the four FUL-like genes in tomato by CRISPR/Cas9-mutagenesis and transcriptome profiling. We demonstrate that sub-functionalization has occurred after duplication within the Solanaceae euFULI and euFULII clades, and that the FUL1 sequence has undergone further divergence during tomato domestication and breeding. FUL2 and MBP20 are highly expressed in the meristem during the vegetative-to-reproductive transition to additively promote tomato flowering and to repress inflorescence branching together with FUL1. Transcriptome analysis in the ful1 ful2 mbp10 mbp20 quadruple mutant revealed that 
the FUL-like genes act parallel to, or downstream of, previously described key regulators such as SFT, FA and AN during both the VM-to-TM transition and the establishment of inflorescence architecture. Instead, our target gene analysis revealed that the delay in transition to flowering may be explained by reduced cytokinin signalling as result of upregulation of CKX genes, while the increased branching is probably caused by delayed FM termination as a result of specific MADS-domain and AP2-like transcription factors that are upregulated in the mutant.

\section{RESULTS}

\section{Expression patterns and protein-protein interaction profiles differ between the tomato FUL homologs}

To investigate to what extent the different FUL-like genes may have overlapping functions in specific organs, we performed expression profiling using qRT-PCR in the cultivar Moneyberg (Figure 1A). The results show that FUL1 and FUL2 are expressed very weakly during vegetative growth and have increased expression in the inflorescence meristem. Their expression remains high throughout reproductive development, where both genes show considerable expression in all floral whorls and all stages of fruit development. FUL2, in particular, is strongly expressed in all floral organs, early fruits and ripening fruits, while FUL 1 expression is moderate until the fruit ripening phase, when it increases strongly as reported previously (Bemer et al., 2012). Our data also show the striking differences in the spatial expression of MBP10 and MBP20 that are both much more weakly expressed than FUL1 and FUL2 in the reproductive tissues, except for the expression of MBP20 in the inflorescence meristem. MBP10 especially was extremely weakly expressed, with detectable levels in stem and flower bud only.

MADS-domain transcription factors regulate multiple developmental processes by forming dimeric complexes (De Folter et al., 2005). To investigate which protein complexes may be formed by the different FUL-like proteins, we performed a yeasttwo hybrid $(\mathrm{Y} 2 \mathrm{H})$ assay to identify the interactions of FUL1, FUL2, MBP10 and MBP20 with other tomato MADS-domain family proteins (Figure 1B, Supplemental 
Figure 1). We chose a set of proteins homologous to MADS-domain proteins known to interact with Arabidopsis FUL (De Folter et al., 2005). While Arabidopsis FUL (AtFUL) can form homodimers (Smaczniak et al., 2012b), none of the tomato FULlike proteins possessed this capacity, nor could they heterodimerize with each other (Supplemental Figure 1). Of the tested MADS-domain proteins, MC, MBP9, MBP12, MBP13, MBP14, MBP22 and MBP24 did not interact with any of the tomato FUL homologs. Our screen showed that the SIFUL proteins could interact with nine other tomato MADS-domain proteins, and we observed clear differences between the interaction profiles of FUL1, FUL2, MBP10 and MBP20. All FUL-like proteins interacted with JOINTLESS (J), J2 (or SIMBP21, hereafter called J2), TM3 and STM3, the co-orthologs of the Arabidopsis flowering integrator SOC1 (Alonge et al., 2020) and the fruit ripening regulator MADS-RIN. FUL2 exhibits the most extensive interaction network, interacting with nine proteins. It is the only protein that strongly interacts with the SEPALLATA (SEP)-like proteins EJ2 and LeSEP1/TM29, and the AGAMOUS (AG) ortholog TAG1 (Figure 1B and Supplemental Figure 1). The latter interaction is especially interesting in the light of the high expression of FUL2 in pistils, where FUL2 may have a specific function in a complex with the co-expressed TAG1. In addition to these specific interactions, FUL2, FUL1 and MBP20 share interactions with the SEP-like protein TM5 and the AGL42-homolog FOREVER YOUNG FLOWER-LIKE (FYFL/SIMBP18), and FUL1 could also weakly interact with EJ2 (Supplemental Figure 1). Part of the interaction pairs we tested has been investigated before in a tomato MADS-domain interaction screen (Leseberg et al., 2008). We could reproduce all the previous results, except for interactions of FUL2 and MBP20 with MBP13, and FUL2 with MBP24. In conclusion, FUL2 can form most protein-protein interactions, which, together with its broad expression pattern, suggests that it can fulfil multiple functions in tomato, similar to FUL in Arabidopsis. FUL1 and MBP20 share a reduced set of interaction partners and MBP10 has the smallest set of interactors. The low number of interactors for MBP10 in combination with its weak overall expression pattern hints at relaxed selective pressure on this gene.

\section{Different variants of the FUL1 gene exist in tomato cultivars}


Upon cloning and sequencing of the FUL1 cDNA of tomato cv. Moneyberg, which was used in our experiments, we noticed a deletion of the $5^{\text {th }}$ base $(\mathrm{G})$ of the last exon as compared to the reference sequence (cv. Heinz). This deletion is predicted to result in a 205 amino acid protein, lacking the C-terminal 40 amino acids as compared to the reference (Figure 1C). Inspection of the genome sequence of 38 resequenced cultivated tomato varieties (Aflitos et al., 2014) showed that approximately half (17) contain this deletion (Supplemental Figure 2A). The latter included the much-studied cv. 'Ailsa Craig'. The deletion was not detected in any of the re-sequenced wild accessions, suggesting that it may have first emerged after domestication. Although the deletion results in a C-terminally truncated protein (FUL1- $\triangle \mathrm{C}$ ), no differences in interactions were observed in a yeast two-hybrid assay (Supplemental figure 2B). An Electrophoretic Mobility Shift Assay (EMSA) also revealed that FUL1- $\triangle \mathrm{C}$ can bind to a CArG-box-containing DNA fragment as a heterotetramer with TM3 (Fig. 1D). Thus, in vitro DNA-binding and protein-protein interaction capacities appear normal for FUL1- $\Delta \mathrm{C}$, indicating, together with the already described fruit ripening function for FUL1- $\Delta \mathrm{C}$ in the cultivars Moneyberg and Ailsa Craig (Wang et al., 2019; Bemer et al., 2012), that the truncated protein is functional. To investigate whether there could be a link between the occurrence of the FUL1- $\triangle C$ allele and certain crop traits, we examined the recently published dataset of Roohanitaziani et al. (2020), in which a wide variety of cultivars and wild species has been characterized for many traits, including pedicel abscission, flowering time, inflorescence architecture, fruit development and fruit ripening. However, we did not find significant differences in any of these traits between the cultivars with a full-length FUL1 allele and those with a truncated allele (Supplemental figure $2 \mathrm{C}$ and $2 \mathrm{D}$ ), suggesting that the occurrence of the FUL1- $\triangle C$ allele does not have a major impact on the investigated features.

\section{FUL2 and MBP20 promote flowering in the primary and sympodial shoots}

To dissect the biological roles of the SIFUL genes in planta, we generated loss-offunction single- and higher-order mutants with the CRISPR/Cas9 method. The first coding exon of each gene was targeted with three single-guide RNAs. After stable tomato transformation of the cultivar Moneyberg, we screened several independent first-generation (TO) transgenic lines for the presence of insertion/deletion (indel) 
alleles by PCR and sequencing. Then we generated the progeny of the primary transgenics (T1) and selected two different homozygous indel alleles for each gene, encoding truncated proteins caused by frameshifts and premature stop codons (Supplemental Figure 3). T2 phenotyping of the selected mutants revealed that the plants with homozygous knockout alleles for either FUL2 or MBP20 exhibited delays in primary shoot flowering, switching to reproductive growth after 13 leaves, compared to 11 in the wild type (Figure 2A). The number of days to first flowering was also significantly decreased, but was more variable between individuals of the same genotype (Supplemental Figure 4A). No significant effect in the number of leaves was observed for the ful1 or mbp10 single mutants (Figure 2A, Supplemental Figure 4B). The observed delay in the transition to flowering was more pronounced in higher-order mutants, with approximately three leaves extra in ful2 mbp20, ful1 ful2 mbp20 and ful1 ful2 mbp10 mbp20 compared to the wild type (Figure 2A). The fact that neither the ful1 nor the mbp10 mutations enhanced the mutant phenotype, suggests that FUL2 and MBP20 are the most important FUL-like genes for promoting the floral transition. In addition to a delay in primary shoot transition, we also observed late flowering in the sympodial shoots of the same set of mutants, increasing to an average of four leaves per sympodial shoot, while the wild type always has three (Figures 2B and 2C, Supplemental Figure 4C and 4D). To further investigate the delayed flowering phenotype, we imaged the primary shoot meristems of the wild type and quadruple mutant at different leaf stages and determined the timing of the reproductive stage transition (Supplemental Figure 4E). In the wild type, meristem transition proceeded rapidly with a visible doming after formation of nine leaves. However, in the quadruple mutant, doming was initiated later and proceeded slower, resulting in completion of the FM only after 12 leaves. In conclusion, FUL2 and MBP20 additively regulate the timing of flowering in both the primary shoot and the sympodial shoots. Later during development, ful1 single mutants also showed an increase in leaf number (Figure 2C, Supplemental Figure 4D), suggesting that FUL1 plays a minor role as well.

\section{FUL1, FUL2 and MBP20 control inflorescence architecture}

We observed that the mutant plants had more branched inflorescences than wildtype plants, which typically produced only non-branched inflorescences (Figure 2D). 
We quantified the branching events for the first seven inflorescences of each plant in the T2 generation. With the exception of mbp10, all mutants showed increased inflorescence complexity, ranging from bi-parous to quintuple-parous inflorescences (Figure 2E). Notably, $13.6 \%$ of the inflorescences from wild-type plants branched, while ful1, ful2 and mbp20 lines produced 43.1\%, 56.1\% and 50\% branched inflorescences, respectively. In higher-order mutants, branching increased further to $\sim 75 \%$ in both the ful 2 mbp20 and the ful1 ful2 mbp20 mutants. mbp10 mutants were hardly branching, similar to the wild type, while mbp10 mbp20 mutants were identical to mbp20 mutants. These results indicate no additional contribution of mbp10 or ful 1 to the branching phenotype of the mutants. Surprisingly, the ful1 mutant did exhibit enhanced branching, but its mutation did not further enhance the phenotype of the ful2 mbp20 mutant, suggesting that FUL1 function depends on FUL2 and/or MBP20 (Supplemental Figure 4F). Interestingly, all first inflorescences did not branch, except for the first inflorescence of ful2 mbp20 (3 plants out of 11) and ful1 ful2 mbp20 (2 plants out of 11). Higher order branching events (i.e. quintuple parous) were only observed in mutant lines where ful2 was included, suggesting that FUL2 has the most prominent role in the repression of inflorescence branching. We quantified the number of flowers on the second to fourth inflorescences. The more complex inflorescences developed more flowers, increasing from on average 10 in wild-type inflorescences to approximately 20 in the higher order mutants (Supplemental Figure $4 \mathrm{G}$ ). To investigate whether the increased branching could also be linked to delayed floral meristem maturation, as observed for the j2 ej2 (Soyk et al., 2017), s and an mutants (Lippmann et al., 2008), we examined different stages of meristem development under the microscope (Figure 2F). We observed delayed FM development of the sympodial shoots, allowing the formation of a second IM before FM determination. The frequency of additional IMs was variable, but most similar to that of the j2 ej2 mutant (Soyk et al., 2017). In conclusion, mutations in FUL2 and MBP20, individually or combined, result in increased branching during inflorescence development. Both genes thus regulate inflorescence architecture in an additive manner, probably by regulating FM determination. FUL1 is also involved in this process as is shown by the ful 1 single mutant phenotype, but its role is masked in higher order mutants that contain ful2 and mbp20 alleles. 


\section{MBP10/20 do not contribute to fruit development and ripening}

FUL1 and FUL2 were reported as redundant regulators of tomato fruit ripening, and FUL2 has an additional function in early fruit development (Bemer et al., 2012; Wang et al., 2019). Since MBP20 is weakly expressed in carpels and early stages of fruit development (Figure 1A), we asked whether it could function in fruit development as well. We therefore examined fruit development and ripening in the different mutant lines. As reported before, the ful2 mutant fruits were smaller with stripes on the pericarp, while the ful1 ful2 mutant fruits were more severely impaired in ripening (Wang et al., 2019). ful2 mbp20 mutant fruits had the same phenotype as ful2 fruits, while triple (ful1 ful2 mbp20) and quadruple mutant fruits resembled ful1 ful2 fruits in terms of width, Brix value, number of locules, pericarp stripes and overall external and internal appearance, indicating that MBP10/20 do not contribute to fruit development and ripening (Supplemental Figures 5 and 6). Remarkable was the high Brix values of fruits that contained ful2 mutant alleles, although this may to a large extent be due to the smaller size of ful2 fruits (Supplemental Figure 5B). Interestingly, the number of locules was slightly, but significantly, enhanced in ful2 single mutants, and in most mutant combinations that contained ful2 (Supplemental Figure 5C and 5D), suggesting that FUL2 is also involved in determining floral meristem size, and thereby carpel number.

\section{Dynamic expression of MADS-box genes in the inflorescence meristem}

The CRISPR mutant analysis revealed that FUL2 and MBP20 promote the transition from vegetative to reproductive development and control inflorescence architecture. To further unveil the role of the tomato FUL-like genes in flowering, we conducted RNA-seq to compare the transcriptome dynamics during three consecutive stages (VM, TM, FM) of meristem development between the wild type and quadruple mutant. For each stage, over 30 meristems from a batch of plants were dissected and pooled for RNA extraction. Three independent batches were grown in the greenhouse at different time points to serve as biological replicates. For practical reasons, the FM and flanking IM were harvested together (further referred to as FM) (see Figure 3A). High-throughput sequencing yielded a minimum of 30M reads per sample. A PCA plot was generated of all 18 samples, which showed clear separation of the VM, TM and FM samples, although there was quite some distance between 
the individual TM samples, probably reflecting the transient nature of this stage (Supplemental Figure 6A). We first determined the expression of the FUL-like genes in the different stages of meristem maturation, revealing dynamic expression changes through the vegetative-to-reproductive transition for FUL1, FUL2 and MBP20 (Figure 3B). FUL2 and MBP20 are already expressed at the VM stage, but their expression highly increased in the TM stage. FUL1 is more weakly expressed in the VM, but also reaches high expression levels in the TM and FM. The higher expression of FUL2 and MBP20 in the VM stage is in line with their prominent role in the determination of flowering time. Of the MADS-box genes encoding SIFUL interactors, $J$ (the SVP-homolog), was highly expressed in all three stages, while the expression of the SEP-like genes EJ2, TM29 and J2 gradually increased from practically absent in VM to clearly expressed in FM. The SOC1-homologs TM3 and STM3 were also expressed in all three stages (Figure $3 \mathrm{C}$ ), but their expression decreased in the FM in contrast to that of EJ2, TM29 and J2. The other potential SIFUL interactors were only weakly expressed. To validate the RNA-seq data analysis, we confirmed the expression patterns of the FUL-like genes, and the genes encoding putative interactors, with qRT-PCR analysis on pooled meristem samples from independently grown batches (Supplemental Figure 7C and 7D).

The combination of the expression and interaction data provides insight into the MADS-domain complexes that may act in planta. Similar to the ful1 ful 2 mbp20 mutants, j2 ej2 mutants also exhibit enhanced branching, in agreement with the increasing expression of all five genes in FMs (Soyk et al., 2017; Park et al., 2012). In contrast, mutations in J, TM3 and STM3 suppress high branching phenotypes, linked to a higher expression in the IM (Figure 3C) (Park et al., 2012; Thouet et al., 2012; Alonge et al., 2020). Thus, FUL1, FUL2 and MBP20 probably form a complex with $\mathrm{J} 2$ and EJ2 in the FM to promote FM maturation. However, the situation is different in the VM, where $J 2$ and EJ2 are not expressed, while TM3, STM3 and $J$ are abundantly present (Figure $3 \mathrm{C}$ ). Both the $j$ and the tm3 stm3 mutants displayed delayed flowering similar to the ful2 mbp20 mutants (Thouet et al., 2012; Alonge et al., 2020), suggesting that FUL2 and MBP20 can physically interact with TM3/STM3 and $\mathrm{J}$ in the VM to induce the transition to flowering. To confirm that these complexes can be formed and bind to CArG-boxes in the DNA, we performed EMSA experiments with FUL2 or MBP20 and the putative interaction partners. Because 
MADS-domain proteins can only bind to the DNA probe as dimers or tetramers (De Folter et al., 2005; Immink et al., 2009), a shifted probe in the assay indicates that a dimer has been formed. Since FUL2 and MBP20 do not form homodimers (Figures $1 \mathrm{~B}$ and 3D, Supplemental Figures $1 \mathrm{~A}$ and 7E), we could in most cases confirm the formation of heterodimeric/tetrameric complexes by the gain of a probe shift. This was only problematic for the interactors that formed strong homodimers themselves (TM3 and J2), but for TM3, the addition of FUL2 or MBP20 resulted in a clear shift towards a tetrameric complex, confirming the yeast two-hybrid data as well (Figure 3D and Supplemental Figure 7E). Thus, based on expression patterns of the genes and interaction capacity, FUL2 and MBP20 probably interact with TM3/STM3 and J in the VM to regulate flowering time, while it is plausible that FUL1, FUL2 and MBP20 form a complex with $\mathrm{J} 2$ and EJ2 to promote FM meristem maturation. In addition, FUL1 and FUL2 may interact with the less abundant TM29 for this purpose.

\section{Identification of Differentially Expressed Genes}

Comparison of WT and quadruple mutant transcriptomes revealed 131 differentially expressed genes (DEGs) for the VM stage (103 up- and 28 down-regulated in the mutant), 162 for the TM stage (137 up- and 25 down-regulated), and 185 DEGs for the FM stage (162 up- and 23 down-regulated), using FDR-corrected p-value $<0.05$ as a threshold for significance and $a \log _{2}$ fold change $>1.0$. These genes significantly overlapped between stages, with 23 genes differentially expressed in all three stages (Supplemental Figure 7B). Many more genes were upregulated in the mutant than there were down-regulated, pointing towards a general repressive function of SIFUL-containing complexes, in agreement with data from Arabidopsis FUL studies (Ferrándiz et al., 2000; Bemer et al., 2017; Balanzà et al., 2018). A large proportion of the DEGs are involved in metabolic processes, such as terpene synthesis or the phenylpropanoid pathway, but the corresponding genes were in general weakly expressed in the meristem (Supplemental Table 1). Notably, the phenylpropanoid pathway is also controlled by FUL1/2 in tomato fruits (Bemer et al., 2012), indicating that the regulation of some identified DEGs is probably of greater importance in other tissues. The DEG lists also contained several interesting genes that are possibly involved in flowering, although previously described tomato key regulators, such as $A N, F A, S F T, S P$ and $S$ were not among the DEGs 
(Supplemental Table 1). We searched the list of DEGs for genes that may explain the flowering phenotypes instead, and identified a few homologs of known Arabidopsis flowering genes, such as VRN1 and AHL15, which are in Arabidopsis involved in the regulation of flowering time and axillary meristem outgrowth, respectively (Levy et al., 2002; Karami et al., 2020a). Also, the MADS-domain factors TM3 and SIMBP13 were significantly upregulated in the quadruple mutant in all three meristem stages. Most interestingly, however, is the identification of four cytokinin signaling genes as targets of the SIFULs.

\section{The tomato FUL-like proteins repress negative regulators of cytokinin signaling}

Compelling evidence shows that cytokinin (CK) is required for SAM activity and FM initiation, and that the interplay of transcription factor regulation and CK signaling controls the size and activity of the shoot apical meristem (Kurakawa et al., 2007; Bartrina et al., 2011; Han et al., 2014). Moreover, a recent report showed that the cytokinin reporter TCSv2 is highly expressed in tomato IM/FM meristems, with a particular high signal in doming meristems (Steiner et al., 2020). In our list of DEGs, we identified several genes involved in CK signaling, namely three CYTOKININ OXIDASEs (CKXS), CKX1/2/6, which degrade bioactive cytokinins, and one type- $A$ ARABIDOPSIS RESPONSE REGULATOR (ARR), ARR16. We verified their differential expression with qRT-PCR in independent samples (Supplemental Figure $8 \mathrm{~A})$. CKX2 and CKX6 were upregulated in the VM and TM stages of the mutant, but not in the FM stage, while $A R R 16$ and $C K X 1$ were only upregulated in the TM stage (Figure 4A). CKXs irreversibly degrade active CKs and type-A ARRs function as negative regulators of the CK response (Brownlee et al., 1975; McGaw and Horgan, 1983; D'Agostino et al., 2000). Therefore, upregulation of $C K X 1 / 2 / 6$ and $A R R 16$ in the VM and TM stages will probably result in a reduced CK content and responsiveness. We further investigated whether FUL2 and MBP20 can directly repress $C K X$ and $A R R$ gene expression by binding to their promoters. We therefore scanned the up- and downstream regions of the $A R R$ and CKX genes for CArG-box motifs, the binding sites for MADS-domain proteins (Kaufmann et al., 2009; Aerts et al., 2018). Putative CArG-boxes were present in all differentially expressed $A R R$ and CKX genes (Supplemental figure 8B). To test whether FUL2 and MBP20 can bind to 
these, we performed EMSAs using fragments containing these CArG-boxes as native probes. Because MADS-domain proteins need to form a dimer to bind to the DNA, we tested TM3-FUL2 and TM3-MBP20 heterodimers, as these proteins form strong heterodimers in yeast and are probably interacting in the VM/TM. In addition, as shown above (Figure 3D), the FUL2/MBP20-TM3 tetrameric complex can be easily distinguished from the TM3 homodimeric complex in EMSA assays. We detected clear shifts for all tested regulatory fragments (Figure 4B), suggesting that the FUL2-TM3 and MBP20-TM3 heterodimers can physically bind to the tested CKX and $A R R$ genes. To investigate whether the CArG-box is essential for the binding, we also generated mutated probes, in which the CArG-box was mildly perturbed by a single-nucleotide mutation in the center of the motifs. This probe mutation abolished or reduced the binding in all cases except for CKX1 (Figure 4B), confirming the importance of the CArG box for the binding of the heterodimers. To determine whether FUL2 and MBP20 both play a role in repressing the cytokinin signalling genes, we harvested meristems from ful2 and mbp20 single mutants and performed qRT-PCRs to determine the upregulation of the $C K X / A R R$ genes. Upregulation was visible in both single mutants, but was in the VM stage more distinct in the ful2 mutant than in the mbp20 mutant, in line with the higher expression of FUL2 at this stage (Figure 3B). In the TM stage, both mutants showed a similar mild upregulation. The upregulation in the single mutants was considerably weaker than in the quadruple mutant, reflecting the partially redundant functions of both genes. These results suggest that both FUL2 and MBP20 directly bind to the promoter of the $C K X 1 / 2 / 6$ and $A R R 16$ genes to repress their expression and thereby upregulate cytokinin signaling in the vegetative meristem at the start of the transition to flowering.

\section{Transcriptome analysis of the first sympodial shoot FM reveals genes possibly involved in the branching phenotype}

Searching for DEGs possibly involved in the flowering phenotype of the quadruple mutant, we found very few genes that could be associated with the inflorescence branching phenotype. However, as mentioned above, the primary shoot FM, which was harvested for the RNA-seq, only rarely gave rise to branched inflorescences. Ubiquitous branching in the quadruple mutant was only observed from the first 
sympodial shoot FM (SFM) onwards. Therefore, we performed an additional RNAseq experiment to compare the transcriptomes of WT and quadruple mutant SFMs (Supplemental Figure 9A). This experiment, with the same set-up as described above, revealed 121 differentially expressed genes (DEGs), of which 96 were upregulated and 25 downregulated in the quadruple mutant. Previously reported key regulators of branching, such as $S, F A$ and $A N$, were not in the list of DEGs. The expression of the $S P$ gene, which suppresses the reproductive transition of the sympodial shoot meristem (Pnueli et al., 1998; Thouet et al., 2008), was remarkable, as it varied considerably between samples. (Supplemental Figure 8B). To identify genes possibly responsible for the inflorescence branching in SFMs, we searched for flowering-related genes that were differentially regulated in the SFM samples, but not in the FM samples (Figure 5A). Four transcription factors were identified that may be involved in the regulation of SFM maturation: APETALA 2b (AP2b) (Karlova et al., 2011), AP2C and the MADS-domain TFS AGAMOUS-LIKE 6 (AGL6) and TM29 (Figure 5B). For TM29, $A P 2 b$ and $A P 2 c$, the differential expression was confirmed with qRT-PCR on independent samples (Supplemental Figure 9C). AP2-like genes are angiosperm-wide regulators of both meristem development and flowering, controlling for example stem-cell maintenance in the Arabidopsis SAM (Würschum et al., 2006) and floral and spikelet meristem initiation/termination in maize (Chuck et al., 2008) in addition to their 'floral' roles in sepal/petal development and repression of the C-function (Yant et al., 2010; Morel et al., 2017). The SEP-like MADS-box gene TM29, which is upregulated in FMs (Figure 3C), has been associated with FM identity control (Ampomah-Dwamena et al., 2002). In addition to these specifically differentially expressed genes, two other genes that are also upregulated in the primary shoot FM, but to a lesser extent (Supplemental Table 1), are probably candidates to explain the branching phenotype as well. Mutation of the first one, TM3, results in reduced inflorescence branching in the ej2 j2 mutant background (Alonge et al., 2020), implying that higher expression of TM3 will cause enhanced branching. The other gene is a close homolog of Arabidopsis $A H L 15$, which suppresses axillary meristem maturation in Arabidopsis (Karami et al., 2020b). If tomato $A H L 15$ is also repressing meristem maturation, this could also contribute to the enhanced branching phenotype. In conclusion, FUL1, FUL2 and MBP20 do not seem to regulate inflorescence branching by modifying the expression of the key 
regulators $S, F A$ or $A N$, but we identified several other downstream transcription factors that may be involved.

\section{FUL1 expression is regulated by FUL2 and MBP20}

Despite the high expression of FUL1 in the TM and FM and the branching phenotype in the ful1 single mutants, the ful1 mutation does not enhance the branching phenotype of the ful2 mbp20 mutants (Figure 2E, Supplemental Figure 4F). The distinct down-regulation of FUL1 in the quadruple mutant may explain this apparent discrepancy (Figure 6A, Supplemental Figure 10A), and indicates that the gene is induced by FUL2, MBP20 and/or by itself via a positive (auto-)regulatory loop. Because the expression of FUL1 is low in the VM, FUL2- and/or MBP20-containing complexes may need to bind to the CArG-boxes in the FUL1 regulatory region to upregulate its expression in TM and FM. To test this and determine the separate effects of FUL1, FUL2 and MBP20 on FUL1 regulation, we performed qRT-PCRs in the corresponding single mutants (Figure 6B). Downregulation was observed in all three single mutants, particularly in the FM stage, but the transcript reduction was most severe in the ful1 mutants. The lower FUL1 mRNA level in the ful1 mutant may be caused by nonsense-mediated mRNA decay (NMD) as a result of the premature stop codon. However, it could also be the result of abolished FUL1 autoregulation, or a combination of both decay and disturbed autoregulation. At this point, we cannot discriminate between these possibilities. It is clear however, that both FUL2 and MBP20 positively regulate FUL1 expression. There are four CArG-boxes in the upstream region of FUL1 that can probably be bound by MADS-domain complexes (Supplemental Figure 10B). To test whether FUL2 and MBP20 can bind, we performed EMSAs with TM3-FUL2 and TM3-MBP20 dimers and observed clear binding to the CArG-box containing probes (Figure 6C), suggesting that FUL1 depends on FUL2 and/or MBP20 for maximal expression in the TM and FM stages.

\section{DISCUSSION}




\section{Subfunctionalization of the tomato FUL-like genes}

Following segmental or whole-genome duplication events, genes with new molecular functions can arise through sub- or neofunctionalization, resulting in divergence of biological functions. We show here that functional divergence also occurred for the tomato FUL-like genes after their multiplication early in the Solanaceae lineage. In addition to their previously described roles in fruit development and ripening (Bemer et al., 2012; Wang et al., 2019), we unveil that FUL1 and FUL2 both regulate IM development, albeit at different levels. MBP20 promotes IM development together with FUL2, but does not contribute to fruit development. We did not observe any phenotype for the mbp10 mutant, nor did the mutation enhance the phenotype in higher-order mutants. This suggests, together with its weak overall expression pattern and low number of protein-protein interactions, that MBP10 may become a pseudogene. In line with this, MBP10 lacks regulatory sequences in its first intron (Maheepala et al., 2019), and has a three amino-acid mutation in the I-domain, a region important for protein-protein interactions (Van Dijk et al., 2010). The loss of MBP10 in other Solanaceae genera such as Petunia also hints in this direction (Maheepala et al., 2019).

Although previous overexpression studies suggested that MBP20 functions in leaf development and FUL2 in stem development and secondary growth (Burko et al., 2013; Wang et al., 2014a; Shalit-Kaneh et al., 2019), we did not observe aberrant phenotypes in these tissues in our knockout mutants. The most probable explanation for this discrepancy is the use of the Cauliflower 355 promoter in the previous experiments (Wang et al., 2014a; Shalit-Kaneh et al., 2019), resulting in ectopic expression and mis-regulation of target genes at a position where FUL2 and MBP20 are usually not expressed. Overexpressing MADS-domain proteins or dominantnegative forms of MADS proteins can also perturb complexes that involve their interaction partners, and the phenotype may thus reflect the phenotype of mutants impaired in other, interacting tomato MADS proteins. However, another possibility is that FUL2 and/or MBP20 function redundantly with other MADS proteins in the investigated tissues. Arabidopsis FUL and SOC1 act redundantly in the regulation of secondary growth (Melzer et al., 2008), and FUL2 may thus function redundantly with (S)TM3 in the tomato stem as well. In conclusion, the four FUL-like genes in tomato underwent a functional divergence during evolution, but together retained 
functions in both inflorescence and fruit development. It is possible that some functions have remained unidentified due to redundancy with other MADS-box genes.

\section{The position of FUL1 in the flower regulatory network}

FUL1 appears to act differently from FUL2 and MBP20 in the inflorescence meristem. It is only weakly expressed in the VM, and its high expression in TM and FM probably depends on FUL2 and MBP20, which are already expressed earlier in the VM and can bind to the FUL1 promoter. (Auto-)regulatory loops are a common phenomenon in MADS-box gene regulation. For example, Arabidopsis AP1 contains a CArG-box in its promoter, which can be bound by its own protein as well as by its paralog $(C A L)$ to achieve high expression levels throughout different stages. (Ye et al., 2016). Because of the delayed induction, FUL1 does not regulate flowering time, but does contribute to meristem maturation and thereby the repression of inflorescence branching.

Interestingly, we found that FUL1 has a premature stop codon at the C-terminus in the cultivar Moneyberg and many other cultivars. Although this truncation does not alter in vivo dimer formation with other MADS-domain proteins (Supplemental Figure 2B), nor disturbs tetramer formation and DNA binding (Figure 1D), the C-terminus may be important for protein activity. It contains the highly conserved, but uncharacterized, FUL-specific PQWML motif (Litt and Irish, 2003). Arabidopsis ful mutants complemented with a FUL copy with a mutation in this motif, were less able to rescue the silique phenotype than those transformed with a wild-type copy, suggesting that the motif is important for protein activity (McCarthy et al., 2015). Interestingly, the truncated allele has not been observed in wild relatives of tomato, and so probably first occurred after domestication (Supplemental Figure 2A). However we did not find a correlation between the presence of the FUL1- $\triangle C$ allele and any trait characterized by Roohanitaziani et al. (2020), but we cannot exclude that the allele has been selected during breeding, for example by conferring slightly larger inflorescences without severe branching.

\section{The role of FUL2 and MBP20 in the tomato flowering network}


Several previously identified tomato flowering genes were revealed to be functional homologs of Arabidopsis flowering genes, such as SFT (FT) and FA (LFY) (Molinero $\square$ Rosales et al., 1999; Molinero-Rosales et al., 2004; Lifschitz et al., 2006), indicating that at least part of the Arabidopsis flowering network is conserved in tomato. However, the knowledge of the regulatory network underlying the tomato sympodial flowering pathway is still fragmented and it is yet unclear whether homologs of many important players in Arabidopsis, such as SOC1 and FLC, are important for tomato flowering as well. Here, we show that tomato FUL-like genes regulate flowering and inflorescence development in tomato, thereby adding a piece to the tomato flowering network puzzle. In Arabidopsis, FUL is a target of FLOWERING LOCUS D (FD) and SQUAMOSA PROMOTER BINDING PROTEINLIKE (SPL) proteins in the photoperiod pathway and the age pathway (Kardailsky et al., 1999; Wang et al., 2009; Jung et al., 2016), and functions partially redundantly with $A P 1$ in the promotion of flowering (Ferrándiz et al., 2000). We demonstrate here that FUL2 and MBP20 additively promote flowering similar to their homolog in Arabidopsis, but it is yet unclear whether they act downstream of SFT and the tomato SPLs as well. However, we did identify putative SPL and FT/FD binding sites in silico in both the FUL2 and MBP20 promoter sequences, suggesting that their expression may be regulated in a similar way. Within our set of DEGs, we did not identify any of the previously identified flowering regulators (e.g. FA, S, SFT, SP), further indicating that the tomato FUL homologs may act downstream of, or parallel to, these factors.

MADS-domain transcription factors bind to the DNA as dimers (De Folter et al., 2005), and since the tomato FUL-like proteins cannot form homodimers, they need to heterodimerize with other MADS-domain proteins to regulate target gene expression. For the regulation of flowering time, FUL2 and MBP20 probably form a complex with TM3, STM3 and J, because the corresponding genes are highly expressed in the vegetative and transition meristem, and both tm3 stm3 and $j$ mutants display a small delay in flowering time (Szymkowiak and Irish, 2006; Alonge et al., 2020) similar to ful2 and mbp20.

Downstream of the FUL-like genes, we discovered several repressors of the cytokinin signaling pathway that are upregulated in the VM and TM stages of the quadruple mutant, probably resulting in reduced CK levels and signaling. In tomato, 
the switch from vegetative to transition meristem is accompanied by cell division in the meristem, which results in the doming of the TM. Steiner et al. (2020) showed that this doming is accompanied by a high cytokinin signal in the meristem. Because CK is a prominent inducer of cell proliferation (Miller et al., 1955), it can probably positively regulate cell division during SAM maturation to allow doming of the meristem and the transition to flowering. In line with this hypothesis, the reduced CK levels may inhibit SAM doming and thereby delay flowering. In Arabidopsis, CK deficiency through overexpression of CKXs diminishes shoot meristem activity and indeed retards flowering (Werner et al., 2003). In addition, initiation of both the axillary meristem and the FM revealed to require a cytokinin signaling pulse (Han et al., 2014; Wang et al., 2014b). Our data suggest that FUL2 and MBP20 may promote flowering through indirect regulation of $\mathrm{CK}$ levels and responsiveness by directly repressing $C K X$ and type-A ARR expression, respectively (Figure 7).

In other species, such as Arabidopsis and Petunia, AP1/FUL-like genes are involved in the establishment of IM/FM identity, and combinatorial mutations result in a loss of identity, leading to a non-flowering phenotype. The Arabidopsis ap1 cal double mutant forms only IMs, because FM identity is lost, and mutation of ful also impairs IM identity, resulting in a more severe vegetative phenotype (Ferrándiz et al., 2000). In Petunia, the four FUL/AP1 genes appear to function redundantly in the establishment of IM/FM identity, and higher order mutants remain for a long time in the vegetative stage (Morel et al., 2019). In tomato, vegetative reversion occurs after a few flowers have formed in mutants of the $A P 1$-homolog $M C$ and the SVP-homolog $J$, suggesting that these are required for FM fate as well (Szymkowiak and Irish, 2006; Thouet et al., 2012). We observed this phenotype only occasionally in the ful mutants, suggesting that the promotion of IM fate is mainly regulated by $J$ and $M C$. It is possible however, that the tomato FUL-like genes act redundantly with $M C$, which would reflect the situation in Petunia.

\section{The role of the SIFULs in the regulation of inflorescence architecture}

We show that loss of function of FUL1, FUL2 and/or MBP20 results in a branched inflorescence that produces an increased number of flowers. The phenotype is variable however, with some mutant inflorescences staying single-parous, while others form up to five branches. Our transcriptome analysis revealed that this 
branching is not caused by regulation of $S, A N$ or $F A$, indicating that they function upstream of, or in parallel with, the SIFUL-containing complexes. Remarkable was the varying expression of the TFL-ortholog $S P$, which may be involved in branching by the maintenance of IM identity. SP seemed higher expressed in the quadruple mutant, but this effect was not significant due to the large variation (Supplemental Figure 7B). However, because the branching phenotype is also highly variable, we cannot exclude that $S P$ is somewhat regulated by the tomato FULs in IM/FM, thereby exerting an effect on the branching phenotype. This would be similar to the repression of the legume TFL-homolog by the euFULII clade proteins VEG1 (pea) or MtFUL1-c (Medicago) (Berbel et al., 2012; Benlloch et al., 2015; Cheng et al., 2018; Zhang et al., 2021).

The branching phenotype of the quadruple mutant is similar to that of the $j 2$ ej2 mutant (Soyk et al., 2017) and our microscopic analysis suggests that it is caused by delayed maturation of the FM as well. Given our in vitro interaction data, which show that the FULs can interact with $\mathrm{J} 2$ and $\mathrm{EJ} 2$, it is plausible that they act together in a complex to promote FM maturation and suppress inflorescence branching. Both the FULs and J2/EJ2 are clearly expressed in the FM in our data (Figure 3C), although our sampling method did not allow a clear distinction between FM and flanking SIM. However, the data from Park et al., (2012) are based on FMs that were completely isolated, and they describe high expression for FUL1 and FUL2 in the FM, while Soyk et al. (2017) describe the same for J2 and EJ2, in agreement with an important role of a FUL1/FUL2/MBP20-J2/EJ2 complex in the regulation of FM maturation. Genetic experiments revealed that the j2 ej2 mutant phenotype is additive to that of $s$, indicating that $J 2$ and EJ2 function separately from the $S$ gene (Soyk et al., 2017), and the same probably accounts for the SIFULs.

Our transcriptome analysis of the SFMs unveiled several genes encoding transcription factors that could function in the regulation of FM maturation downstream of the SIFULs (see Figure 7). Two of these, TM29 and TM3, are also encoding for MADS-domain transcription factors, which are upregulated in the quadruple mutant FMs. In particular TM3 is interesting, because its expression is high in VM and TM, but drops in FM. This suggests that TM3 is repressing FM maturation, in line with the observation that the tm3 stm3 double mutants represses the enhanced branching phenotype of $j 2$ ej2. The upregulation of TM3 will thus delay 
FM maturation and thereby enhance branching. Indications for the involvement of the other genes, $A H L 15, A P 2 b$ and $A P 2 c$, rather comes from research in Arabidopsis and maize. In Arabidopsis, $A H L 15$ is repressing meristem maturation in the axillary buds (Karami et al., 2020a), while AP2-like genes regulate meristem development in both Arabidopsis and maize (Würschum et al., 2006; Chuck et al., 2008). The role of $A P 2 b$ and $A P 2 c$ could also be linked to their role as A-class floral organ genes, because AP2-like genes are in many species involved in the repression of the Cfunction (Morel et al., 2017), of which the onset marks complete FM maturation. Which of these downstream factors is most important for the increased branching phenotype still needs to be determined with future genetic experiments and localization studies to determine what their function is in either the FM or the IM.

\section{MATERIALS AND METHODS}

\section{Accession numbers}

FUL1, Solyc06g069430; FUL2, Solyc03g114830; MBP10, Solyc02g065730; MBP20, Solyc02g089210; J, Solyc11g010570; J2, Solyc12g038510; EJ2, Solyc03g114840; TM29, Solyc02g089200; MADS-RIN, Solyc05g012020; TM5, Solyc05g015750; TM3, Solyc01g093965; STM3, Solyc01g092950; SIMBP18, Solyc03g006830; TAG1, Solyc02g071730; SIMBP24, Solyc01g105800; SIMBP13， Solyc08g080100; SIMBP14, Solyc12g056460; SIMBP9, Solyc04g076680; SIMBP12, Solyc12g088090; SIMBP22, Solyc11g005120; MADS-MC, Solyc05g056620; AHL15, Solyc12g087950; AP2b, Solyc02g064960; AP2c, Solyc02g093150; AGL6, Solyc01g093960; CKX1, Solyc04g016430; CKX2, Solyc10g017990; CKX6, Solyc12g008900; ARR16, Solyc06g048930; AP2b, Solyc02g064960; Ap2c, Solyc02g093150. Supplementary Table 1 contains the accession numbers of the DEGs.

\section{Plant materials and growing conditions.}

Tomato cv. Moneyberg was used for the Agrobacterium tumefaciens-mediated transformation experiments (Van Roekel et al., 1993). Tissue culture was conducted in a growth chamber with $16 \mathrm{~h}$ light and $8 \mathrm{~h}$ dark at $25^{\circ} \mathrm{C}$. After rooting, the 
transformed plants were cultivated in a $21^{\circ} \mathrm{C}$ growth chamber (16h light/8h dark). 25-day old plants were moved to the greenhouse and grown under ambient temperatures and natural light, supplemented with artificial sodium lights.

\section{qRT-PCR analysis.}

For qRT-PCR analysis of SIFUL expression, root, shoot, leaves, flower organs, and fruits of different stages were harvested from wild-type tomato plants. RNA was extracted with a CTAB/LiCl method (Porebski et al., 1997), DNase treated with Ambion Turbo DNase (AM1907) and cDNA was synthesized with the iScript cDNA synthesis kit (Bio-Rad). Real-time RT-PCR was performed with the iQ SYBR Green Supermix from Bio-Rad with a standard 2-step program of 40 cycles, annealing at 60 ${ }^{0} \mathrm{C}$. Primer efficiencies were tested beforehand and only primer pairs with equal efficiencies were compared (all primer sequences, including reference primers, are listed in Supplemental Table 2).

\section{Yeast two-hybrid}

Protein-protein interaction assays in yeast were performed using the GAL4 System using Gateway vectors as described (De Folter and Immink, 2011). The coding sequences for bait proteins and prey proteins were cloned into the pDEST32 and pDEST22 vectors respectively, and the vectors were transformed into the PJ69-4A and PJ69-4a yeast strains. The interaction screen was performed using -LWH dropout medium, supplemented with $3 \mathrm{mM}$ 3-amino-1,2,4-triazole (3-AT) or -LWA dropout medium. Plates were incubated for 5 days at RT. All primer sequences used for cloning are listed in Supplemental Table 2.

\section{CRISPR construct generation and stable tomato transformation}

The ful 2 and ful $1 / 2$ transgenic CRISPR lines have been previously generated (Wang et al., 2019). The constructs for all other lines were generated using GoldenGate cloning and the MoClo toolkit according to (Weber et al., 2011). Briefly, each gRNA was fused to the synthetic U6 promoter as U6p::gRNA, and ligated in a Level 1 vector. Level 1 constructs pICH47732-NOSpro::NPTII::OCST, pICH4774235S::Cas9::NOST, pICH47751-35S::GFP::ter35S, pICH47761-gRNA1, pICH47772gRNA2, plCH47781-gRNA3 and the linker plCH41822 were cut/ligated into the level 
2 vector pICSL4723 as described. After confirming the constructs, the plasmids were transformed into Agrobacterium strain C58C1. All primers are listed in Supplemental Table 2. The above constructs were introduced into tomato cv Moneyberg by Agrobacterium tumefaciens-mediated transformation. Homozygous T1 or T2 transgenic plants were used for phenotypic and molecular characterization.

\section{Meristem imaging}

Shoot apices were dissected from young plants using a forceps and older leaf primordia were removed to expose meristems under the stereomicroscope. Immediately after dissection, live meristems were imaged using a euromex scientific camera.

\section{Meristem transcriptome profiling}

The domesticated tomato (S. lycopersicum) cultivar Moneyberg and the homozygous ful1 ful2 mbp10 mbp20 mutant generated in the Moneyberg background were used for transcriptome profiling. For each biological replicate sample, a batch of plants was grown and from each plant, the primary shoot meristem was harvested, either in the VM, TM or FM stage. For the SFM samples, the first FM from the sympodial shoot was harvested. About 60 plants were grown per batch (to harvest $>30$ meristems). All stages were harvested in triplicate for both the wild type and quadruple mutant plants. The batches for the different replicates were grown in the greenhouse sequentially. Meristems were dissected using a stereoscope, and tissue was processed for RNA stabilization using an acetone fixation technique (Park et al., 2012). RNA was extracted using the PicoPure RNA Extraction kit (Arcturus). More than 30 meristems were collected for each sample, yielding 1 3 $\mu \mathrm{g}$ RNA, which was enriched for mRNA and processed into cDNA libraries using the Illumina TruSeq Stranded Total RNA LT Sample Prep Kit (Illumina). After quality control (Qubit and Fragment Analyzer), samples were sequenced using Illumina NovaSeq 2x150 nt Paired End sequencing. Samples were randomized across sequencing flow cells and lanes within flow cells. After quality control, all data were analyzed using the CLC work package. The raw data has been deposited in GEO under accession number GSE154419. For data validation, new batches of plants were grown and 
processed as described above, and the samples were analysed using qRT-PCR analysis (see Supplemental Table 2 for the primers).

\section{Electrophoretic mobility shift assays (EMSAs)}

FUL2 and MBP20 coding sequences were amplified from wild-type Moneyberg cDNA and cloned into pSPUTK (see Supplemental Table 2 for all primer sequences). The pSPUTK promoter allowed in vitro protein synthesis using the TnT® SP6 High-Yield Wheat Germ Protein Expression System (Promega) according to the manufacturer's instructions. The probe fragments consisted of a region of 80$100 \mathrm{bp}$ with the canonical CArG-box in the middle, and were amplified from genomic DNA; The mutated probe fragments were generated by overlapping PCR using primers that replaced one base pair in the middle of the CArG-box. EMSAs were performed essentially as described by Smaczniak et al. (2012) with minor modifications. Oligonucleotides were fluorescently labelled using DY-682. Labelling was performed by PCR using vector-specific DY-682-labelled primers followed by PCR purification with NucleoSpin® Gel and PCR Clean-up kit (MACHEREYNAGEL). Gel shifts were visualized using a LiCor Odyssey imaging system at 700 $\mathrm{nm}$.

\section{ACKNOWLEDGMENTS}

We would like to thank Stuart Jansma, Albert van der Veen, Janne Hageman, Siye Chen and Iris Zahn for their helpful work on yeast two-hybrid analysis, tomato transformation or meristem harvesting. We greatly appreciate Geurt Versteeg and Teus van den Brink for their help in taking care of tomato plants and collecting seeds in the greenhouse, and André Maassen and Michiel Lammers for their efforts to create enough greenhouse space. This work has been supported by a grant from the Dutch Scientific Organization (NWO) (ALWOP.199) to M.B., a fellowship from the China Scholarship Council (CSC) to X.J., a fellowship from CAPES and CAPES/Nuffic (BEX 7686/13-7) to G.L. and the Fundação de Amparo à Pesquisa do Estado de São Paulo (FAPESP process 2010/52012-4) and Coordenação de Aperfeiçoamento de Pessoal de Nível Superior (CAPES/NUFFIC-BEX 0256/13-7) to J.H.L. 


\section{AUTHOR CONTRIBUTIONS}

M.B. conceived the project and designed the experiments; X.J. performed the qPCRs, tomato CRISPR/cas9 mutagenesis, RNA-seq experiments and EMSAs; K.R, G.L. and J.H.L. did the Yeast Two Hybrid analyses; V.V. measured the fruit phenotypes; R.A.d.M. analyzed FUL1 alleles in cultivars and assisted with the RNAseq analysis; G.C.A. and M.B. supervised the project; M.B. and X.J analyzed the data, prepared the figures and wrote the article. All authors read and approved the final version.

\section{REFERENCES}

Aerts, N., de Bruijn, S., van Mourik, H., Angenent, G.C., and van Dijk, A.D. (2018). Comparative analysis of binding patterns of MADS-domain proteins in Arabidopsis thaliana. BMC plant biology 18, 131.

Aflitos, S., Schijlen, E., de Jong, H., de Ridder, D., Smit, S., Finkers, R., Wang, J., Zhang, G., Li, N.e.a., and Consortium, T.G.S. (2014). Exploring genetic variation in the tomato (Solanum section Lycopersicon) clade by whole-genome sequencing. The Plant Journal 80, 136-148.

Alonge, M., Wang, X., Benoit, M., Soyk, S., Pereira, L., Zhang, L., Suresh, H., Ramakrishnan, S., Maumus, F., and Ciren, D. (2020). Major Impacts of Widespread Structural Variation on Gene Expression and Crop Improvement in Tomato. Cell.

Ampomah-Dwamena, C., Morris, B.A., Sutherland, P., Veit, B., and Yao, J.-L. (2002). Downregulation of TM29, a tomato SEPALLATA homolog, causes parthenocarpic fruit development and floral reversion. Plant physiology 130, 605-617.

Balanzà, V., Martínez-Fernández, I., Sato, S., Yanofsky, M.F., Kaufmann, K., Angenent, G.C., Bemer, M., and Ferrándiz, C. (2018). Genetic control of meristem arrest and life span in Arabidopsis by a FRUITFULL-APETALA2 pathway. Nature communications $9,565$.

Bartrina, I., Otto, E., Strnad, M., Werner, T., and Schmülling, T. (2011). Cytokinin regulates the activity of reproductive meristems, flower organ size, ovule formation, and thus seed yield in Arabidopsis thaliana. The Plant Cell 23, 69-80.

Bemer, M., van Mourik, H., Muiño, J.M., Ferrándiz, C., Kaufmann, K., and Angenent, G.C. (2017). FRUITFULL controls SAUR10 expression and regulates Arabidopsis growth and architecture. Journal of experimental botany 68, 3391-3403.

Bemer, M., Karlova, R., Ballester, A.R., Tikunov, Y.M., Bovy, A.G., Wolters-Arts, M., de Barros Rossetto, P., Angenent, G.C., and de Maagd, R.A. (2012). The tomato FRUITFULL homologs TDR4/FUL1 and MBP7/FUL2 regulate ethylene-independent aspects of fruit ripening. The Plant Cell 24, 4437-4451.

Benlloch, R., Berbel, A., Ali, L., Gohari, G., Millán, T., and Madueño, F. (2015). Genetic control of inflorescence architecture in legumes. Frontiers in plant science 6, 543-543.

Berbel, A., Ferrándiz, C., Hecht, V., Dalmais, M., Lund, O.S., Sussmilch, F.C., Taylor, S.A., Bendahmane, A., Ellis, T.H.N., Beltrán, J.P., Weller, J.L., and Madueño, F. (2012). VEGETATIVE1 is essential for development of the compound inflorescence in pea. Nature Communications 3, 797. 
Bowman, J.L., Alvarez, J., Weigel, D., Meyerowitz, E.M., and Smyth, D.R. (1993). Control of flower development in Arabidopsis thaliana by APETALA1 and interacting genes. Development 119, 721-743.

Brownlee, B.G., Hall, R.H., and Whitty, C.D. (1975). 3-Methyl-2-butenal: An Enzymatic Degradation Product of the Cytokinin, N 6-( $\Delta 2$-Isopentenyl) adenine. Canadian Journal of Biochemistry 53, 37-41.

Burko, Y., Shleizer-Burko, S., Yanai, O., Shwartz, I., Zelnik, I.D., Jacob-Hirsch, J., Kela, I., EshedWilliams, L., and Ori, N. (2013). A role for APETALA1/fruitfull transcription factors in tomato leaf development. The Plant Cell 25, 2070-2083.

Chae, E., Tan, Q.K.-G., Hill, T.A., and Irish, V.F. (2008). An Arabidopsis F-box protein acts as a transcriptional co-factor to regulate floral development. Development 135, 1235-1245.

Cheng, X., Li, G., Tang, Y., and Wen, J. (2018). Dissection of genetic regulation of compound inflorescence development in Medicago truncatula. Development 145, dev158766.

Chuck, G., Meeley, R., and Hake, S. (2008). Floral meristem initiation and meristem cell fate are regulated by the maize \&lt;em\&gt;AP2\&lt;/em\&gt; genes \&lt;em\&gt;ids1\&lt;/em\&gt; and \&lt;em\&gt;sid1\&lt;/em\&gt. Development 135, 3013.

D'Agostino, I.B., Deruere, J., and Kieber, J.J. (2000). Characterization of the response of the Arabidopsis response regulator gene family to cytokinin. Plant physiology 124, 1706-1717.

De Folter, S., and Immink, R.G. (2011). Yeast protein-protein interaction assays and screens. In Plant Transcription Factors (Springer), pp. 145-165.

De Folter, S., Immink, R.G., Kieffer, M., Pařenicová, L., Henz, S.R., Weigel, D., Busscher, M., Kooiker, M., Colombo, L., and Kater, M.M. (2005). Comprehensive interaction map of the Arabidopsis MADS box transcription factors. The Plant Cell 17, 1424-1433.

Ferrándiz, C., Gu, Q., Martienssen, R., and Yanofsky, M.F. (2000). Redundant regulation of meristem identity and plant architecture by FRUITFULL, APETALA1 and CAULIFLOWER. Development 127, 725-734.

Gao, R., Wang, Y., Gruber, M.Y., and Hannoufa, A. (2018). miR156/SPL10 modulates lateral root development, branching and leaf morphology in Arabidopsis by silencing AGAMOUS-LIKE 79. Frontiers in plant science 8, 2226.

Gomez Roldan, M.V., Périlleux, C., Morin, H., Huerga-Fernandez, S., Latrasse, D., Benhamed, M., and Bendahmane, A. (2017). Natural and induced loss of function mutations in SIMBP21 MADS-box gene led to jointless-2 phenotype in tomato. Scientific reports 7, 4402.

Goslin, K., Zheng, B., Serrano-Mislata, A., Rae, L., Ryan, P.T., Kwaśniewska, K., Thomson, B., Ó'Maoiléidigh, D.S., Madueño, F., Wellmer, F., and Graciet, E. (2017). Transcription Factor Interplay between LEAFY and APETALA1/CAULIFLOWER during Floral Initiation. Plant physiology 174, 1097-1109.

Gu, Q., Ferrándiz, C., Yanofsky, M.F., and Martienssen, R. (1998). The FRUITFULL MADS-box gene mediates cell differentiation during Arabidopsis fruit development. Development 125, 15091517.

Han, Y., Zhang, C., Yang, H., and Jiao, Y. (2014). Cytokinin pathway mediates APETALA1 function in the establishment of determinate floral meristems in Arabidopsis. Proceedings of the National Academy of Sciences 111, 6840-6845.

Immink, R.G., Tonaco, I.A., de Folter, S., Shchennikova, A., van Dijk, A.D., Busscher-Lange, J., Borst, J.W., and Angenent, G.C. (2009). SEPALLATA3: the'glue'for MADS box transcription factor complex formation. Genome biology 10, R24.

Jaakola, L., Poole, M., Jones, M.O., Kämäräinen-Karppinen, T., Koskimäki, J.J., Hohtola, A., Häggman, H., Fraser, P.D., Manning, K., and King, G.J. (2010). A SQUAMOSA MADS box gene involved in the regulation of anthocyanin accumulation in bilberry fruits. Plant Physiology 153, 1619-1629.

Jaudal, M., Zhang, L., Che, C., and Putterill, J. (2015). Three Medicago MtFUL genes have distinct and overlapping expression patterns during vegetative and reproductive development and 
35S:MtFULb accelerates flowering and causes a terminal flower phenotype in Arabidopsis. Front Genet 6, 50-50.

Jia, Z., Jiang, B., Gao, X., Yue, Y., Fei, Z., Sun, H., Wu, C., Sun, S., Hou, W., and Han, T. (2015). GmFULa, a FRUITFULL homolog, functions in the flowering and maturation of soybean. Plant cell reports 34, 121-132.

Jung, J.-H., Lee, H.-J., Ryu, J.Y., and Park, C.-M. (2016). SPL3/4/5 integrate developmental aging and photoperiodic signals into the FT-FD module in Arabidopsis flowering. Molecular plant 9, 1647-1659.

Karami, O., Rahimi, A., Khan, M., Bemer, M., Hazarika, R.R., Mak, P., Compier, M., van Noort, V., and Offringa, R. (2020a). A suppressor of axillary meristem maturation promotes longevity in flowering plants. Nature Plants 6, 368-376.

Karami, O., Rahimi, A., Khan, M., Bemer, M., Hazarika, R.R., Mak, P., Compier, M., van Noort, V., and Offringa, R. (2020b). A suppressor of axillary meristem maturation promotes longevity in flowering plants. bioRxiv.

Kardailsky, I., Shukla, V.K., Ahn, J.H., Dagenais, N., Christensen, S.K., Nguyen, J.T., Chory, J., Harrison, M.J., and Weigel, D. (1999). Activation tagging of the floral inducer FT. Science 286, 1962-1965.

Karlova, R., Rosin, F.M., Busscher-Lange, J., Parapunova, V., Do, P.T., Fernie, A.R., Fraser, P.D., Baxter, C., Angenent, G.C., and de Maagd, R.A. (2011). Transcriptome and metabolite profiling show that APETALA2a is a major regulator of tomato fruit ripening. The Plant cell 23, 923-941.

Kaufmann, K., Muino, J.M., Jauregui, R., Airoldi, C.A., Smaczniak, C., Krajewski, P., and Angenent, G.C. (2009). Target genes of the MADS transcription factor SEPALLATA3: integration of developmental and hormonal pathways in the Arabidopsis flower. PLoS biology 7.

Kaufmann, K., Wellmer, F., Muiño, J.M., Ferrier, T., Wuest, S.E., Kumar, V., Serrano-Mislata, A., Madueno, F., Krajewski, P., and Meyerowitz, E.M. (2010). Orchestration of floral initiation by APETALA1. science 328, 85-89.

Kurakawa, T., Ueda, N., Maekawa, M., Kobayashi, K., Kojima, M., Nagato, Y., Sakakibara, H., and Kyozuka, J. (2007). Direct control of shoot meristem activity by a cytokinin-activating enzyme. Nature 445, 652-655.

Lee, J., and Lee, I. (2010). Regulation and function of SOC1, a flowering pathway integrator. Journal of Experimental Botany 61, 2247-2254.

Leseberg, C.H., Eissler, C.L., Wang, X., Johns, M.A., Duvall, M.R., and Mao, L. (2008). Interaction study of MADS-domain proteins in tomato. Journal of experimental botany 59, 2253-2265.

Levy, Y.Y., Mesnage, S., Mylne, J.S., Gendall, A.R., and Dean, C. (2002). Multiple roles of Arabidopsis VRN1 in vernalization and flowering time control. Science 297, 243-246.

Li, C., Lin, H., Chen, A., Lau, M., Jernstedt, J., and Dubcovsky, J. (2019). Wheat VRN1 and FUL2 play critical and redundant roles in spikelet meristem identity and spike determinacy. bioRxiv, 510388.

Lifschitz, E., Eviatar, T., Rozman, A., Shalit, A., Goldshmidt, A., Amsellem, Z., Alvarez, J.P., and Eshed, Y. (2006). The tomato FT ortholog triggers systemic signals that regulate growth and flowering and substitute for diverse environmental stimuli. Proceedings of the National Academy of Sciences 103, 6398-6403.

Lippman, Z.B., Cohen, O., Alvarez, J.P., Abu-Abied, M., Pekker, I., Paran, I., Eshed, Y., and Zamir, D. (2008). The making of a compound inflorescence in tomato and related nightshades. PLoS biology 6, e288.

Litt, A., and Irish, V.F. (2003). Duplication and diversification in the APETALA1/FRUITFULL floral homeotic gene lineage: implications for the evolution of floral development. Genetics 165, 821-833. 
Liu, C., Teo, Z.W.N., Bi, Y., Song, S., Xi, W., Yang, X., Yin, Z., and Yu, H. (2013). A conserved genetic pathway determines inflorescence architecture in Arabidopsis and rice. Developmental cell 24, 612-622.

Liu, D., Wang, D., Qin, Z., Zhang, D., Yin, L., Wu, L., Colasanti, J., Li, A., and Mao, L. (2014). The SEPALLATA MADS-box protein SLMBP 21 forms protein complexes with JOINTLESS and MACROCALYX as a transcription activator for development of the tomato flower abscission zone. The Plant Journal 77, 284-296.

MacAlister, C.A., Park, S.J., Jiang, K., Marcel, F., Bendahmane, A., Izkovich, Y., Eshed, Y., and Lippman, Z.B. (2012). Synchronization of the flowering transition by the tomato TERMINATING FLOWER gene. Nature genetics 44, 1393.

Maheepala, D.C., Emerling, C.A., Rajewski, A., Macon, J., Strahl, M., Pabón-Mora, N., and Litt, A. (2019). Evolution and Diversification of FRUITFULL Genes in Solanaceae. Frontiers in plant science 10, 43.

Mandel, M.A., Gustafson-Brown, C., Savidge, B., and Yanofsky, M.F. (1992). Molecular characterization of the Arabidopsis floral homeotic gene APETALA1. Nature 360, 273.

McCarthy, E.W., Mohamed, A., and Litt, A. (2015). Functional divergence of APETALA1 and FRUITFULL is due to changes in both regulation and coding sequence. Frontiers in Plant Science 6, 1076.

McGarry, R.C., and Ayre, B.G. (2012). Manipulating plant architecture with members of the CETS gene family. Plant Science 188-189, 71-81.

McGaw, B.A., and Horgan, R. (1983). Cytokinin catabolism and cytokinin oxidase. Phytochemistry 22, 1103-1105.

Melzer, S., Lens, F., Gennen, J., Vanneste, S., Rohde, A., and Beeckman, T. (2008). Flowering-time genes modulate meristem determinacy and growth form in Arabidopsis thaliana. Nature genetics 40, 1489.

Miller, C.O., Skoog, F., Von Saltza, M.H., and Strong, F. (1955). Kinetin, a cell division factor from deoxyribonucleic acid1. Journal of the American Chemical Society 77, 1392-1392.

Molinero-Rosales, N., Latorre, A., Jamilena, M., and Lozano, R. (2004). SINGLE FLOWER TRUSS regulates the transition and maintenance of flowering in tomato. Planta 218, 427-434.

Molinero-Rosales, N., Jamilena, M., Zurita, S., Gómez, P., Capel, J., and Lozano, R. (1999). FALSIFLORA, the tomato orthologue of FLORICAULA and LEAFY, controls flowering time and floral meristem identity. The Plant Journal 20, 685-693.

Morel, P., Heijmans, K., Rozier, F., Zethof, J., Chamot, S., Bento, S.R., Vialette-Guiraud, A., Chambrier, P., Trehin, C., and Vandenbussche, M. (2017). Divergence of the Floral AFunction between an Asterid and a Rosid Species. The Plant cell 29, 1605-1621.

Morel, P., Chambrier, P., Boltz, V., Chamot, S., Rozier, F., Bento, S.R., Trehin, C., Monniaux, M., Zethof, J., and Vandenbussche, M. (2019). Divergent Functional Diversification Patterns in the SEP/AGL6/AP1 MADS-box Transcription Factor Superclade. The Plant Cell 31, 3033-3056.

Nakano, T., Kimbara, J., Fujisawa, M., Kitagawa, M., Ihashi, N., Maeda, H., Kasumi, T., and Ito, Y. (2012). MACROCALYX and JOINTLESS interact in the transcriptional regulation of tomato fruit abscission zone development. Plant physiology 158, 439-450.

Pabón-Mora, N., Ambrose, B.A., and Litt, A. (2012). Poppy APETALA1/FRUITFULL orthologs control flowering time, branching, perianth identity, and fruit development. Plant physiology 158, 1685-1704.

Pabón-Mora, N., Sharma, B., Holappa, L.D., Kramer, E.M., and Litt, A. (2013). The A quilegia FRUITFULL-like genes play key roles in leaf morphogenesis and inflorescence development. The Plant Journal 74, 197-212. 
Park, S.J., Jiang, K., Schatz, M.C., and Lippman, Z.B. (2012). Rate of meristem maturation determines inflorescence architecture in tomato. Proceedings of the National Academy of Sciences 109, 639-644.

Périlleux, C., Bouché, F., Randoux, M., and Orman-Ligeza, B. (2019). Turning Meristems into Fortresses. Trends in Plant Science 24, 431-442.

Ping, J., Liu, Y., Sun, L., Zhao, M., Li, Y., She, M., Sui, Y., Lin, F., Liu, X., Tang, Z., Nguyen, H., Tian, Z., Qiu, L., Nelson, R.L., Clemente, T.E., Specht, J.E., and Ma, J. (2014). Dt2 is a gain-of-function MADS-domain factor gene that specifies semideterminacy in soybean. The Plant cell 26, 2831-2842.

Pnueli, L., Carmel-Goren, L., Hareven, D., Gutfinger, T., Alvarez, J., Ganal, M., Zamir, D., and Lifschitz, E. (1998). The SELF-PRUNING gene of tomato regulates vegetative to reproductive switching of sympodial meristems and is the ortholog of CEN and TFL1. Development 125, 1979-1989.

Porebski, S., Bailey, L.G., and Baum, B.R. (1997). Modification of a CTAB DNA extraction protocol for plants containing high polysaccharide and polyphenol components. Plant molecular biology reporter 15, 8-15.

Roohanitaziani, R., de Maagd, R.A., Lammers, M., Molthoff, J., Meijer-Dekens, F., van Kaauwen, M.P.W., Finkers, R., Tikunov, Y., Visser, R.G.F., and Bovy, A.G. (2020). Exploration of a Resequenced Tomato Core Collection for Phenotypic and Genotypic Variation in Plant Growth and Fruit Quality Traits. Genes 11.

Sablowski, R. (2007). Flowering and determinacy in Arabidopsis. Journal of Experimental Botany 58, 899-907.

Serrano-Mislata, A., Goslin, K., Zheng, B., Rae, L., Wellmer, F., Graciet, E., and Madueño, F. (2017). Regulatory interplay between LEAFY, APETALA1/CAULIFLOWER and TERMINAL FLOWER1: New insights into an old relationship. Plant Signal Behav 12, e1370164-e1370164.

Shalit-Kaneh, A., Eviatar-Ribak, T., Horev, G., Suss, N., Aloni, R., Eshed, Y., and Lifschitz, E. (2019). The flowering hormone florigen accelerates secondary cell wall biogenesis to harmonize vascular maturation with reproductive development. Proceedings of the National Academy of Sciences 116, 16127-16136.

Shima, Y., Kitagawa, M., Fujisawa, M., Nakano, T., Kato, H., Kimbara, J., Kasumi, T., and Ito, Y. (2013). Tomato FRUITFULL homologues act in fruit ripening via forming MADS-box transcription factor complexes with RIN. Plant Molecular Biology 82, 427-438.

Smaczniak, C., Immink, R.G., Angenent, G.C., and Kaufmann, K. (2012a). Developmental and evolutionary diversity of plant MADS-domain factors: insights from recent studies. Development 139, 3081-3098.

Smaczniak, C., Immink, R.G., Muiño, J.M., Blanvillain, R., Busscher, M., Busscher-Lange, J., Dinh, Q.P., Liu, S., Westphal, A.H., and Boeren, S. (2012b). Characterization of MADS-domain transcription factor complexes in Arabidopsis flower development. Proceedings of the National Academy of Sciences 109, 1560-1565.

Souer, E., Rebocho, A.B., Bliek, M., Kusters, E., de Bruin, R.A., and Koes, R. (2008). Patterning of inflorescences and flowers by the F-Box protein DOUBLE TOP and the LEAFY homolog ABERRANT LEAF AND FLOWER of petunia. The Plant Cell 20, 2033-2048.

Soyk, S., Lemmon, Z.H., Sedlazeck, F.J., Jiménez-Gómez, J.M., Alonge, M., Hutton, S.F., Van Eck, J., Schatz, M.C., and Lippman, Z.B. (2019). Duplication of a domestication locus neutralized a cryptic variant that caused a breeding barrier in tomato. Nature plants 5, 471.

Soyk, S., Lemmon, Z.H., Oved, M., Fisher, J., Liberatore, K.L., Park, S.J., Goren, A., Jiang, K., Ramos, A., and van der Knaap, E. (2017). Bypassing negative epistasis on yield in tomato imposed by a domestication gene. Cell 169, 1142-1155. e1112.

Steiner, E., Israeli, A., Gupta, R., Shwartz, I., Nir, I., Leibman-Markus, M., Tal, L., Farber, M., Amsalem, Z., Ori, N., Müller, B., and Bar, M. (2020). Characterization of the cytokinin sensor TCSv2 in arabidopsis and tomato. Plant Methods 16, 152-152. 
Szymkowiak, E.J., and Irish, E.E. (2006). JOINTLESS suppresses sympodial identity in inflorescence meristems of tomato. Planta 223, 646-658.

Theissen, G., and Saedler, H. (2001). Plant biology: floral quartets. Nature 409, 469.

Thouet, J., Quinet, M., Ormenese, S., Kinet, J.-M., and Périlleux, C. (2008). Revisiting the involvement of SELF-PRUNING in the sympodial growth of tomato. Plant physiology 148, 6164.

Thouet, J., Quinet, M., Lutts, S., Kinet, J.-M., and Périlleux, C. (2012). Repression of floral meristem fate is crucial in shaping tomato inflorescence. PLoS One 7.

Van Dijk, A.D., Morabito, G., Fiers, M., van Ham, R.C., Angenent, G.C., and Immink, R.G. (2010). Sequence motifs in MADS transcription factors responsible for specificity and diversification of protein-protein interaction. PLoS computational biology 6, e1001017.

Van Roekel, J.S., Damm, B., Melchers, L.S., and Hoekema, A. (1993). Factors influencing transformation frequency of tomato (Lycopersicon esculentum). Plant cell reports 12, 644647.

Wang, J.-W., Czech, B., and Weigel, D. (2009). miR156-regulated SPL transcription factors define an endogenous flowering pathway in Arabidopsis thaliana. Cell 138, 738-749.

Wang, R., da Rocha Tavano, E.C., Lammers, M., Martinelli, A.P., Angenent, G.C., and de Maagd, R.A. (2019). Re-evaluation of transcription factor function in tomato fruit development and ripening with CRISPR/Cas9-mutagenesis. Scientific reports $9,1696$.

Wang, S., Lu, G., Hou, Z., Luo, Z., Wang, T., Li, H., Zhang, J., and Ye, Z. (2014a). Members of the tomato FRUITFULL MADS-box family regulate style abscission and fruit ripening. Journal of experimental botany 65, 3005-3014.

Wang, Y., Wang, J., Shi, B., Yu, T., Qi, J., Meyerowitz, E.M., and Jiao, Y. (2014b). The stem cell niche in leaf axils is established by auxin and cytokinin in Arabidopsis. The Plant Cell 26, 20552067.

Weber, E., Gruetzner, R., Werner, S., Engler, C., and Marillonnet, S. (2011). Assembly of designer TAL effectors by Golden Gate cloning. PloS one 6.

Werner, T., Motyka, V., Laucou, V., Smets, R., Van Onckelen, H., and Schmülling, T. (2003). Cytokinin-deficient transgenic Arabidopsis plants show multiple developmental alterations indicating opposite functions of cytokinins in the regulation of shoot and root meristem activity. The Plant Cell 15, 2532-2550.

Würschum, T., Gross-Hardt, R., and Laux, T. (2006). APETALA2 regulates the stem cell niche in the Arabidopsis shoot meristem. The Plant cell 18, 295-307.

Yant, L., Mathieu, J., Dinh, T.T., Ott, F., Lanz, C., Wollmann, H., Chen, X., and Schmid, M. (2010). Orchestration of the Floral Transition and Floral Development in \&lt;em\&gt;Arabidopsis\&lt;/em\&gt; by the Bifunctional Transcription Factor APETALA2. The Plant Cell 22, 2156.

Ye, L., Wang, B., Zhang, W., Shan, H., and Kong, H. (2016). Gains and losses of cis-regulatory elements led to divergence of the Arabidopsis APETALA1 and CAULIFLOWER duplicate genes in the time, space, and level of expression and regulation of one paralog by the other. Plant physiology 171, 1055-1069.

Yuste-Lisbona, F.J., Quinet, M., Fernández-Lozano, A., Pineda, B., Moreno, V., Angosto, T., and Lozano, R. (2016). Characterization of vegetative inflorescence (mc-vin) mutant provides new insight into the role of MACROCALYX in regulating inflorescence development of tomato. Scientific reports 6, 18796.

Zhang, P., Wang, R., Wang, X., Mysore, K.S., Wen, J., Meng, Y., Gu, X., Niu, L., and Lin, H. (2021). MtFULc controls inflorescence development by directly repressing MtTFL1 in Medicago truncatula. Journal of Plant Physiology 256, 153329.

Zhao, J., Jiang, L., Che, G., Pan, Y., Li, Y., Hou, Y., Zhao, W., Zhong, Y., Ding, L., and Yan, S. (2019). A Functional Allele of CSFUL1 Regulates Fruit Length through Repressing CSSUP and Inhibiting Auxin Transport in Cucumber. The Plant Cell 31, 1289-1307. 
Zhu, Y., Klasfeld, S., Jeong, C.W., Jin, R., Goto, K., Yamaguchi, N., and Wagner, D. (2020). TERMINAL FLOWER 1-FD complex target genes and competition with FLOWERING LOCUS T. Nature Communications 11, 5118. 


\section{FIGURES}
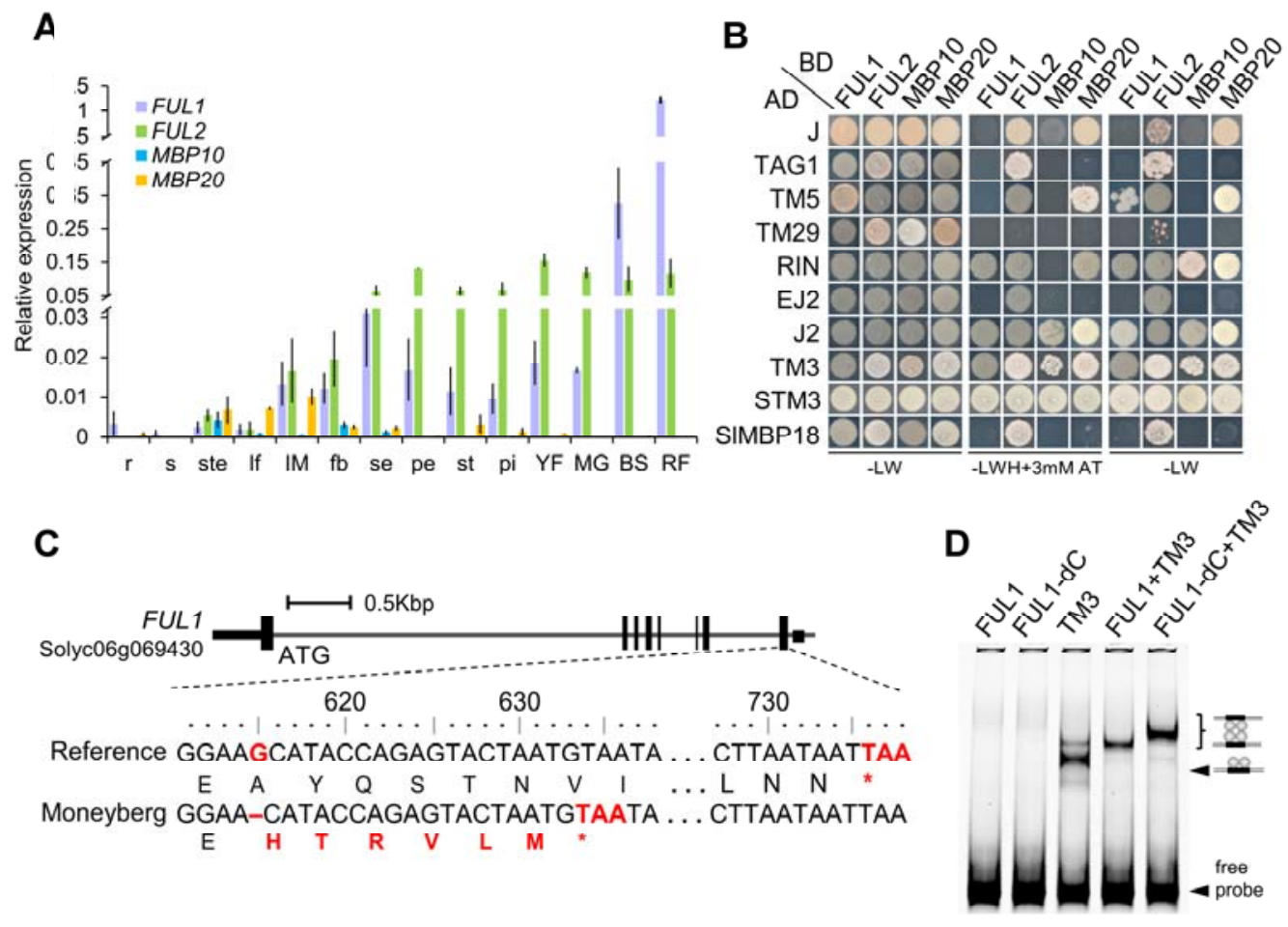

Figure 1. Characterization of the tomato FUL-like genes. (A) Relative expression profiles of SIFUL genes in different organs obtained by qRT-PCR. r: two-week-old root; s: two-week-old shoot; ste: stem below apex; If: young leaf; IM: inflorescence meristem (dissected apex); fb: closed flower bud; se: sepal; pe: petal; st: stamen; pi: pistil; YF: young fruit; MG: mature green fruit; BR: breaker stage fruit; RR: red ripe fruit. The error bars indicate $\pm S D$ based on three biological replicates. (B) Yeast twohybrid assays showing the protein interactions of the FUL-like proteins with other tomato MADS-domain proteins. L, leucine; W, tryptophan; $\mathrm{H}$, histidine; A, adenine; 3AT, 3-amino-1,2,4-triazole. (C) The position of the one-nucleotide deletion present at the 3 ' end of the FUL1 gene in the cultivar Moneyberg, resulting in a protein lacking the C-terminus. The red letters indicate the differences in amino acids, the red nucleotides indicate the stop codons, the vertical black bars are exons. The dotted line refers to what is expanded in the sequence below (part of the last exon). (D) 
EMSA assays showing that the FUL1 truncated protein (FUL1- $\triangle \mathrm{C}$ ) can form higherorder complexes in vitro.
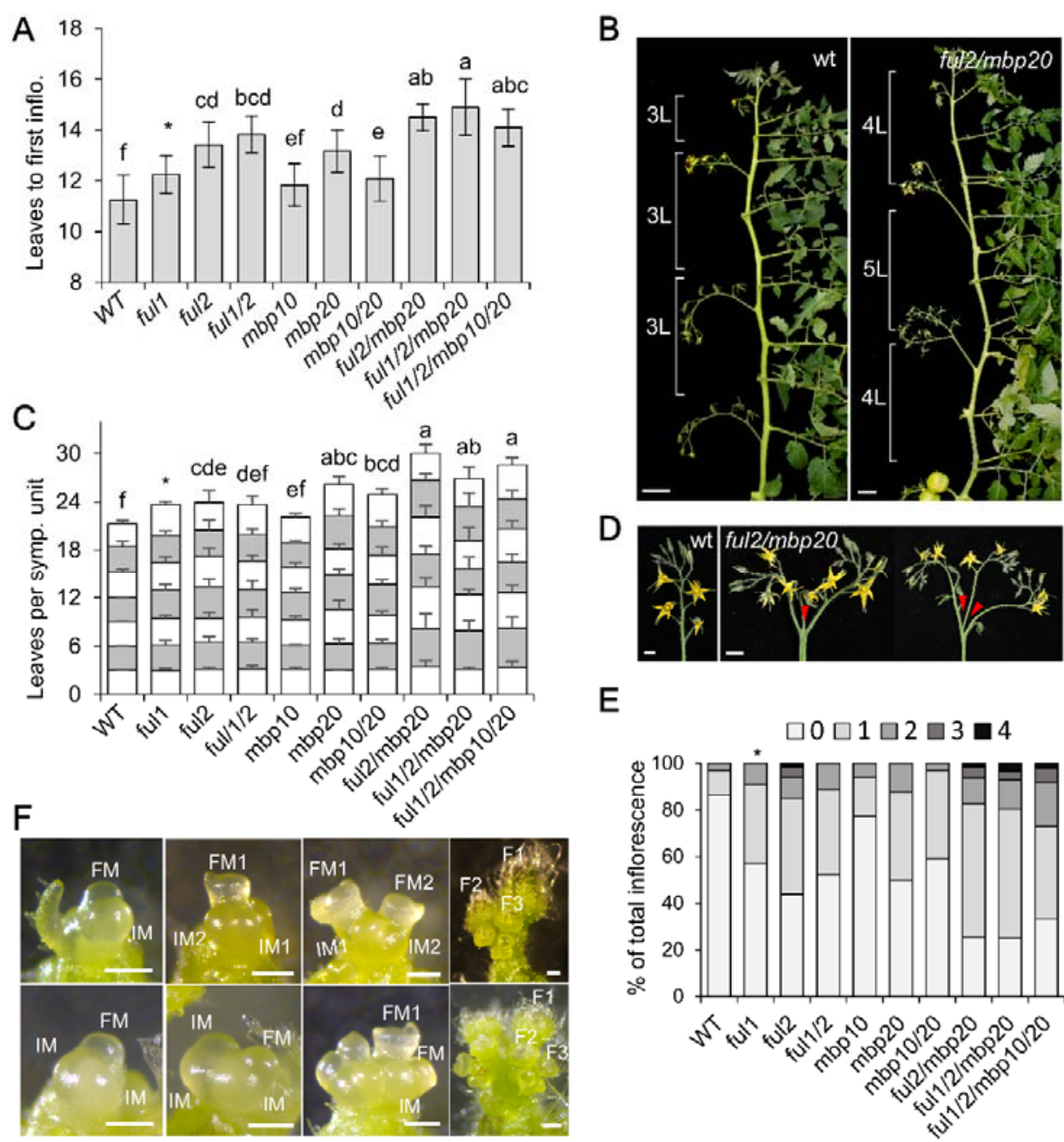

E

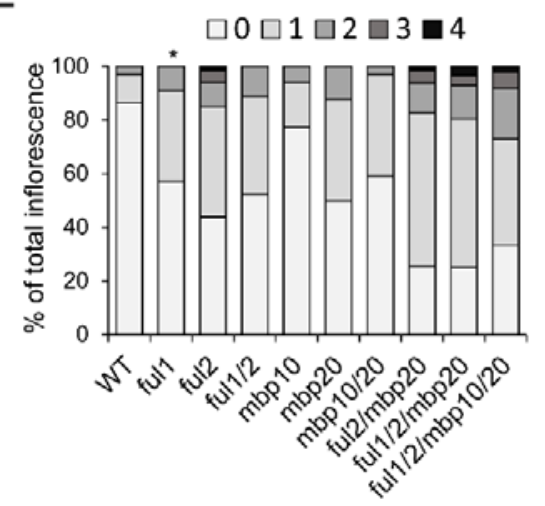

Figure 2. Disruption of the FUL-like genes results in delayed flowering and enhanced inflorescence branching. (A) Quantification of primary shoot flowering for wild-type (WT) and slful mutants. (B) Representative sympodial shoots from WT plants and the ful2 mbp20 mutant. L: leaf; Scale bars: $5 \mathrm{~cm}$. (C) Cumulative numbers of leaves in successive sympodial units for the first seven units in WT and slful mutants. The sum of the leaves was used to test the significance. (D) Representative images of wild type and mutant (branched) inflorescences. Red arrowheads indicate branching events. Scale bars: $2 \mathrm{~cm}$. (E) Proportion of branched inflorescences in the indicated genotypes. (F) A developmental series of sympodial meristems of WT (upper) and quadruple mutant (lower panel) from the FM stage to an inflorescence. 
FM: floral meristem; IM: inflorescence meristem; F: flower. White bar: $200 \mu \mathrm{m}$. In A and $C$, mean values $( \pm S D$ ) were compared between genotypes using one-way ANOVA followed by a post hoc LSD test, different letters indicate the difference at $\mathrm{P}<0.05$ level, six individual T2 offspring plants were analyzed per line and the data from the two different genotypes were combined for each mutant (e.g. $2 \times 6$ individuals for ful1 etc.). The asterisk in A,C and $E$ indicates that the ful1 data is acquired from a second phenotyping experiment and normalized against a wild type control.

A
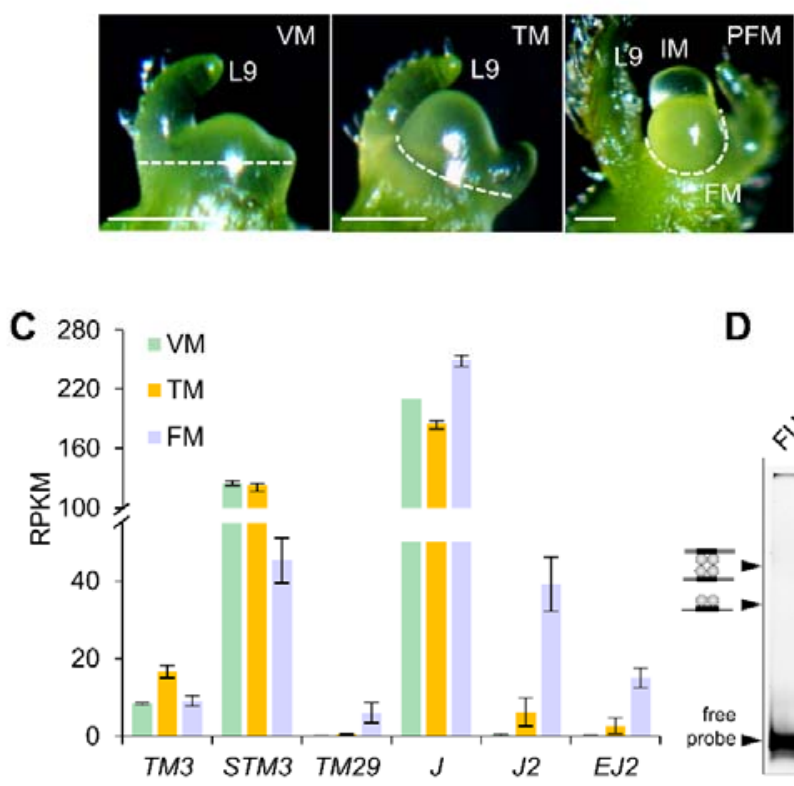

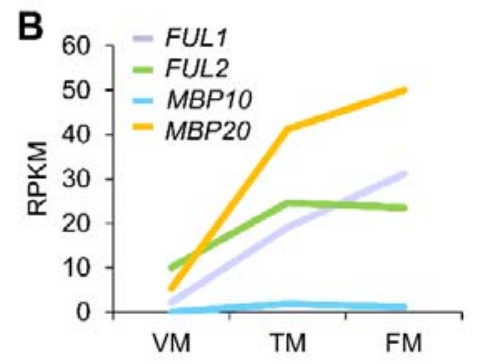

Figure 3. Gene expression dynamics in the primary shoot meristem. (A) Manual microdissection of the three successive meristem stages of primary shoot meristems for transcriptome profiling. Dashed lines indicate the dissected tissues. White bar: $100 \mu \mathrm{m}$. PFM: primay floral meristem. (B), (C) Normalized gene expression (RPKM) of the FUL-like genes and TM3, STM3, TM29, J, J2, and EJ2 in wild-type meristem stages. The values shown (mean $\pm \mathrm{SD}$ ) are the average of three replicates. (D) 
EMSA showing FUL2 interactions with MADS-domain proteins. VM: vegetative meristem; TM: transition meristem; FM: floral meristem.

A
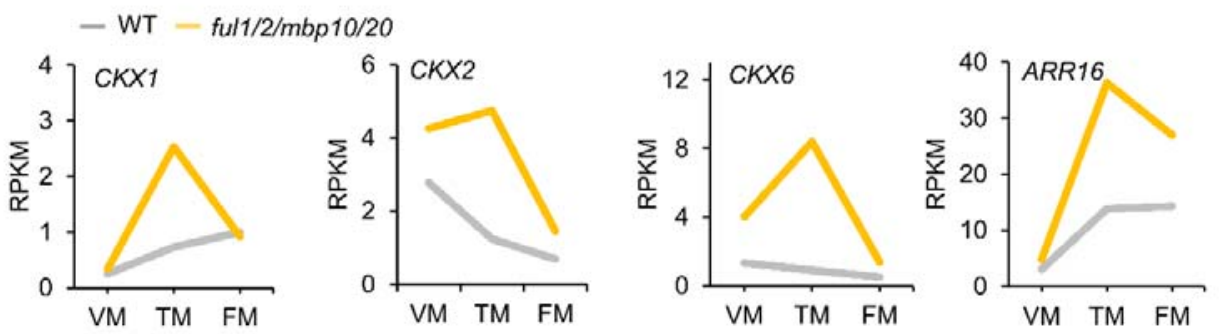

B
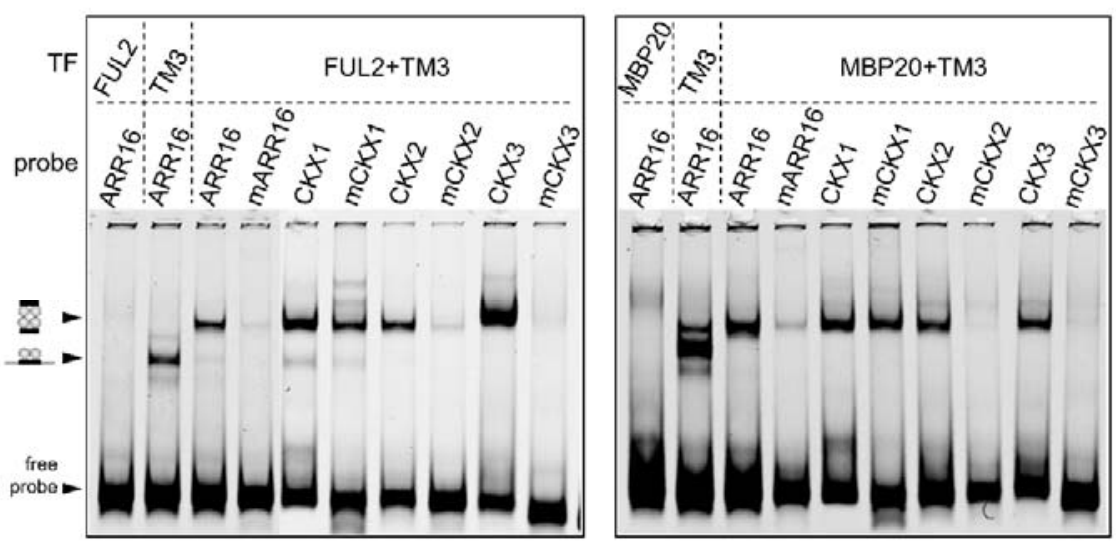

C
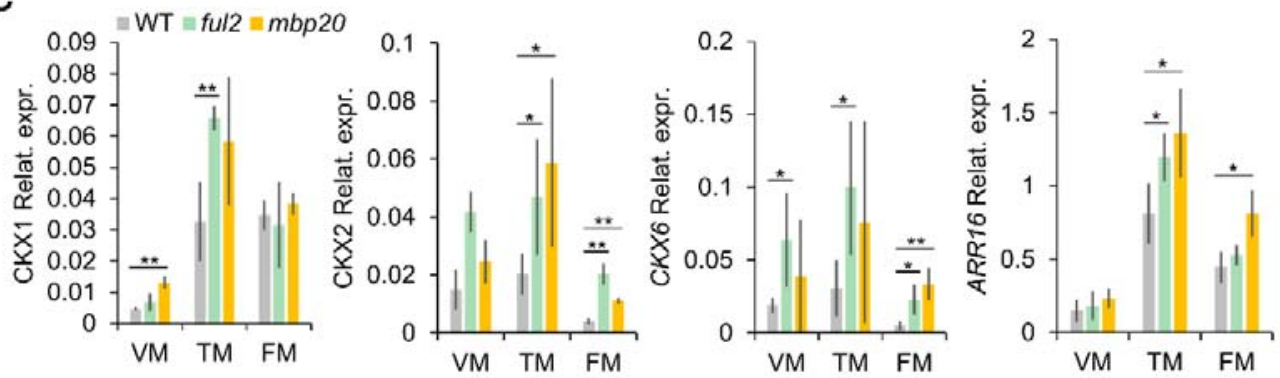

Figure 4. FUL2 and MBP20 regulate $C K X 1 / 2 / 6$ and $A R R 16$ expression. (A) Normalized gene expression (RPKM) for CKX1/2/6 and ARR16 across vegetative and reproductive meristem stages. (B) EMSA showing that FUL2-TM3 directly binds to promoter fragments of $C K X 1 / 2 / 6$ and $A R R 16$ in vitro. The arrow indicates the shift of the probe caused by the binding of FUL2-TM3. The mutated versions of the promoter fragments are indicated with an ' $m$ '. TF: transcription factor. (C) Expression of $C K X 1 / 2 / 6$ and $A R R 16$ during SAM transition in wild-type, ful2 and mbp20 obtained by qRT-PCR. Significant differences were calculated using one-tailed 
Student's t test $\left({ }^{*}, \leq 0.05\right.$ and $\left.{ }^{* *}, P \leq 0.01\right)$. The values shown (mean $\left.\pm S D\right)$ are the average of three replicates. VM: vegetative meristem; TM: transition meristem; FM: floral meristem.
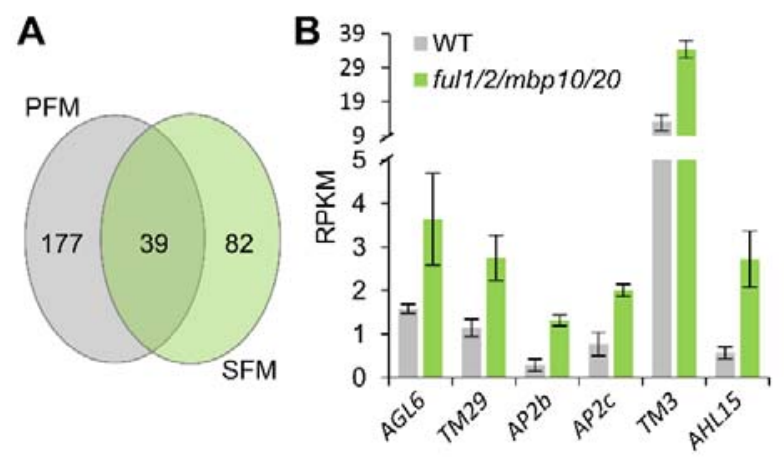

Figure 5. Gene expression in floral meristems of the sympodial shoot in WT and slful quadruple mutants. (A) Venn diagram showing the overlap of differentially expressed genes (DEGs) in the floral meristem of primary shoot (PFM) and sympodial shoot (SFM) of wild-type (WT) and the quadruple mutant. (B) Normalized gene expression (RPKM) of DEGs of interest in WT SFM. The values shown (mean $\pm \mathrm{SD}$ ) are the average of three biological replicates. 

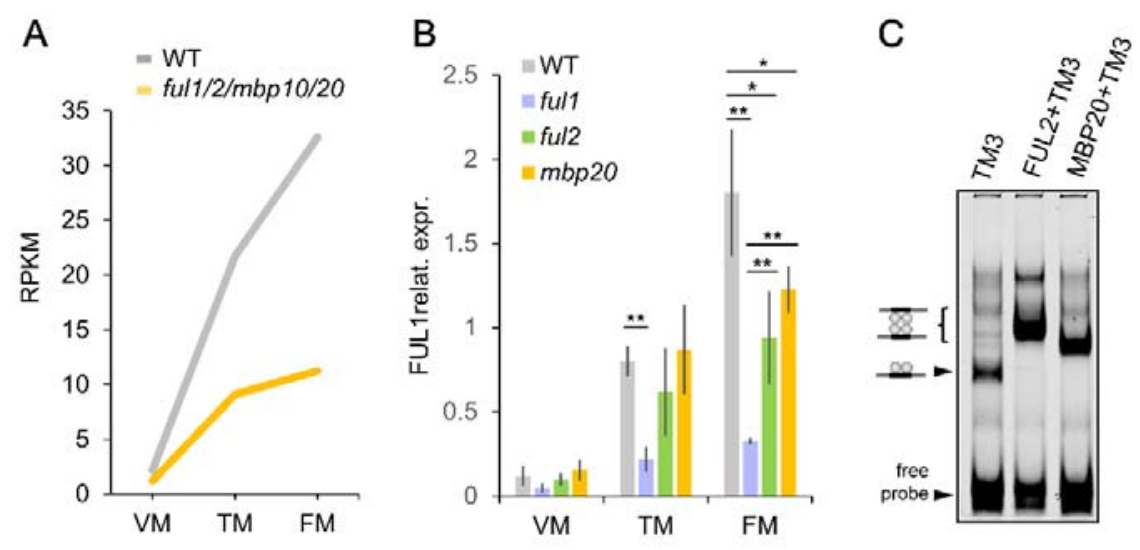

Figure 6. FUL2 and MBP20 regulate FUL1 expression. (A) Normalized gene expression (RPKM) of FUL1 across vegetative and reproductive meristem stages in wild type (WT) and quadruple mutant. (B) FUL1 expression during SAM transition in WT, ful1, ful2 and mbp20 tested by qRT-PCR. (C) EMSA showing that FUL2/TM3 and MBP20/TM3 directly bind to the promoters of FUL1 in vitro. Average and standard deviation of two independent replicates per stage. The arrow indicates the shift of the probe caused by the binding of FUL2-TM3 or MBP20/TM3. The values shown (mean $\pm S D$ ) are the average of three biological replicates. Significant differences were calculated using one-tailed Student's $t$ test $\left({ }^{*}, \leq 0.05\right.$ and ${ }^{* *}, \mathrm{P} \leq$ 0.01). VM: vegetative meristem; TM: transition meristem; FM: floral meristem. 


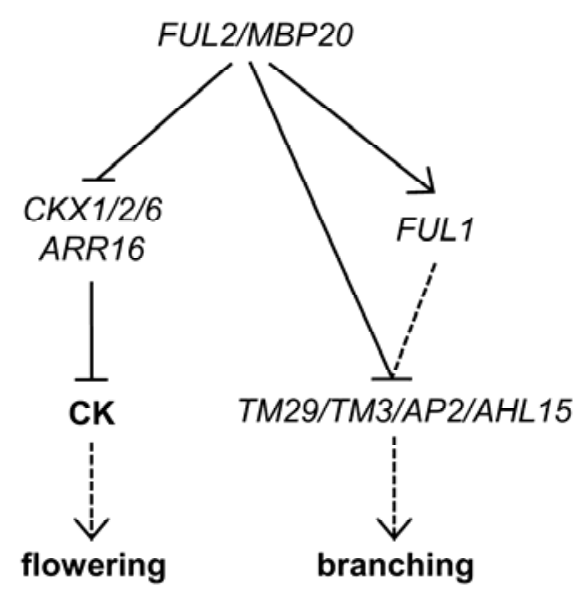

Figure 7. Model of SIFUL regulation of flowering time and inflorescence branching in tomato. The connections between the different regulators are based on the results of this study and other work described in the text. Solid lines display confirmed interactions, while dashed lines represent putative interactions. CK is cytokinin. 